\title{
General Mechanism of Osmolytes' Influence on Protein Stability Irrespective of the Type of Osmolyte Cosolvent
}

\author{
Aneta Panuszko, Piotr Bruździak, Emilia Kaczkowska, Janusz Stangret \\ Department of Physical Chemistry, Chemical Faculty, Gdansk University of Technology \\ Narutowicza 11/12, 80-233 Gdansk, Poland
}

\section{Table of contents:}

S1. ATR-FTIR spectroscopy: determination of the protein-affected osmolyte spectra.

S1.1. The method

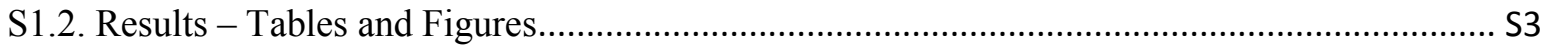

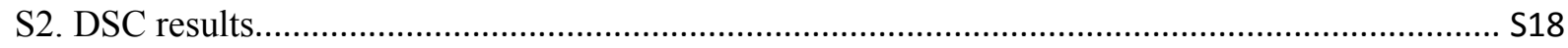

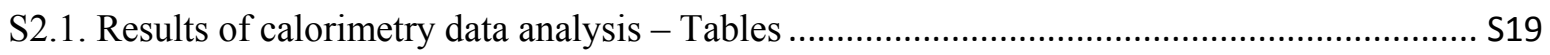

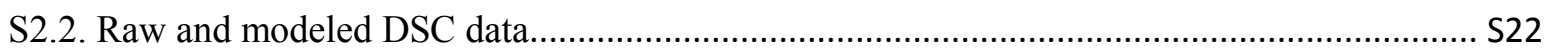

S3. Difference HDO spectra method: determination and interpretation of the solute - affected water

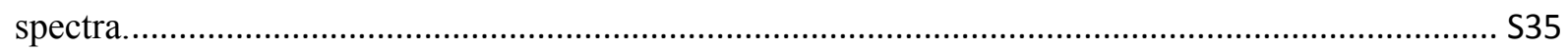

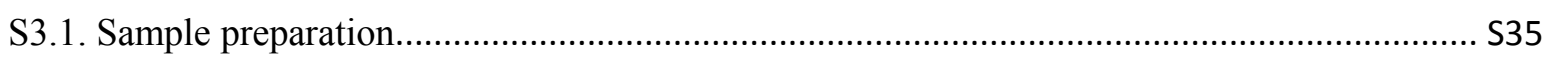

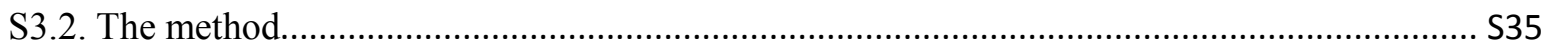

S3.3. Determination of the osmolyte-affected water spectra..................................................... S37

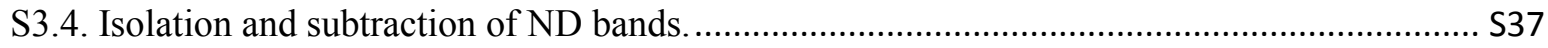

S3.5. Structural and energetic characterization of water affected by osmolytes........................... S39

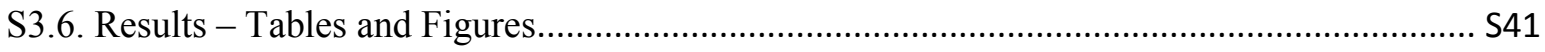




\section{S1. ATR-FTIR spectroscopy: determination of the protein-affected osmolyte spectra.}

\section{S1.1. The method}

All solutions of a osmolyte-water-protein system were prepared by weight. Molar concentrations and molalities, necessary to subtract various spectra and to isolate appropriate affected spectra, were calculated with densities determined by means of DSA 5000 densitometer (Anton-Paar) and by assuming that partial volume of the lysozyme in a solution was equal to $0.703 \mathrm{~cm}^{3} / \mathrm{g}$. Scheme $\mathrm{S} 1$ represents an overall scheme of solutions preparation and indicates most important series of spectra used to determine various properties of analyzed systems.

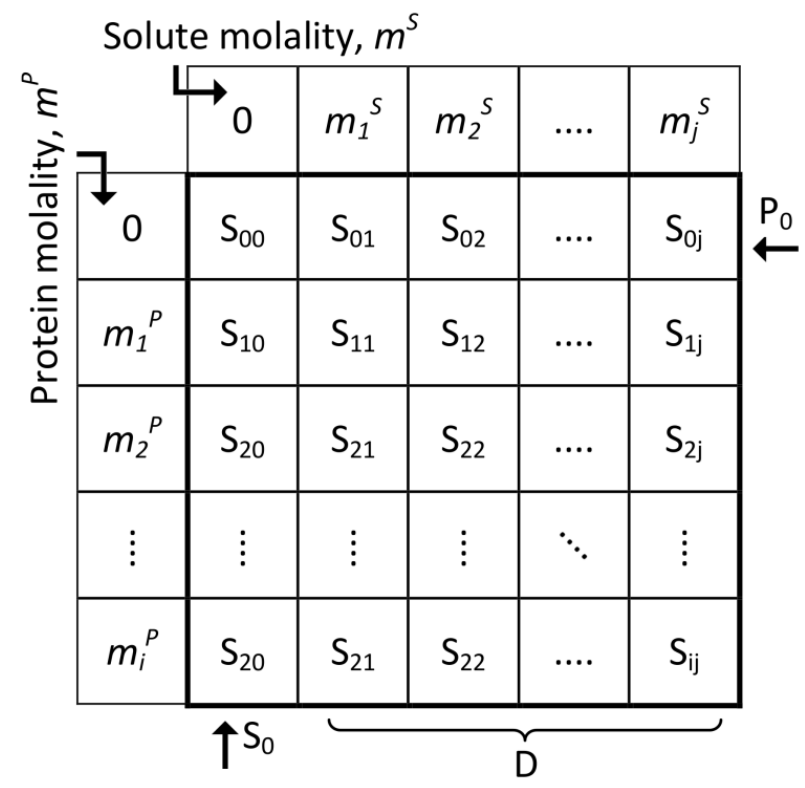

Scheme S1. Matrix of spectra necessary to determine the influence of protein on solute. $\mathrm{m}_{\mathrm{i}}^{\mathrm{P}}-$ protein molality $(\mathrm{mol} / \mathrm{kg}) ; \mathrm{m}_{\mathrm{j}}{ }^{\mathrm{S}}$ - solute molality $(\mathrm{mol} / \mathrm{kg}) ; \mathrm{S}_{\mathrm{ij}}$ - spectrum of the solute-waterprotein system, where molalities of all constituents vary. $0^{\text {th }}$ series are composed only of two constituents (water and solute or water and protein). In each column series of spectra $\mathrm{m}^{\mathrm{S}}=$ const.; $\mathrm{P}_{0}-$ spectra of the $0^{\text {th }}$ row allow to calculate the solute spectrum extrapolated to $\mathrm{m}_{\mathrm{S}}=0$ $\mathrm{mol} / \mathrm{kg}$ and the spectrum of solute affected by its concentration change; $\mathrm{S}_{0}-$ spectra of the $0^{\text {th }}$ 
column allow to determine pure protein spectra of various molalities, which later on can be used to interpolate an appropriate spectrum of the protein to be subtracted from a spectrum of a solute-water-protein system. The proper molality of the protein (an of any other constituent) in a sample is determined on the basis of weighted amounts of each constituents in the sample. ; D - each column series of spectra allow to determine a spectrum of solute affected by protein presence at a given solute molality; a series of determined affected spectra of solute at different molalities can serve to interpolate an affected spectrum at $1 \mathrm{~mol} / \mathrm{kg}$ of solute; such a spectrum can be easily compared with similar spectra of other solutes, thus it serves as a reference affected spectrum of solute.

\section{S1.2. Results - Tables and Figures}

Table S1. Molalities ( $\mathrm{mol} / \mathrm{kg}$ ) of urea and its derivatives

\begin{tabular}{ccccc}
\hline Solute & $m_{1}$ & $m_{2}$ & $m_{3}$ & $m_{4}$ \\
\hline $\mathrm{U}$ & 0.9842 & 2.2487 & 3.0621 & 4.0158 \\
$\mathrm{NMU}$ & 0.5004 & 1.0020 & 1.5204 & 1.9997 \\
$\mathrm{NEU}$ & 0.5032 & 1.0104 & 1.5274 & 2.0622 \\
$\mathrm{NBU}$ & 0.1520 & 0.3000 & 0.5045 & 0.7031 \\
$\mathrm{DMU}$ & 0.3004 & 0.5039 & 1.0099 & - \\
$\mathrm{DEU}$ & 0.5096 & 0.9983 & 1.5067 & 2.0084 \\
TMU & 0.4854 & 0.9843 & 2.7193 & 2.9617 \\
\hline
\end{tabular}




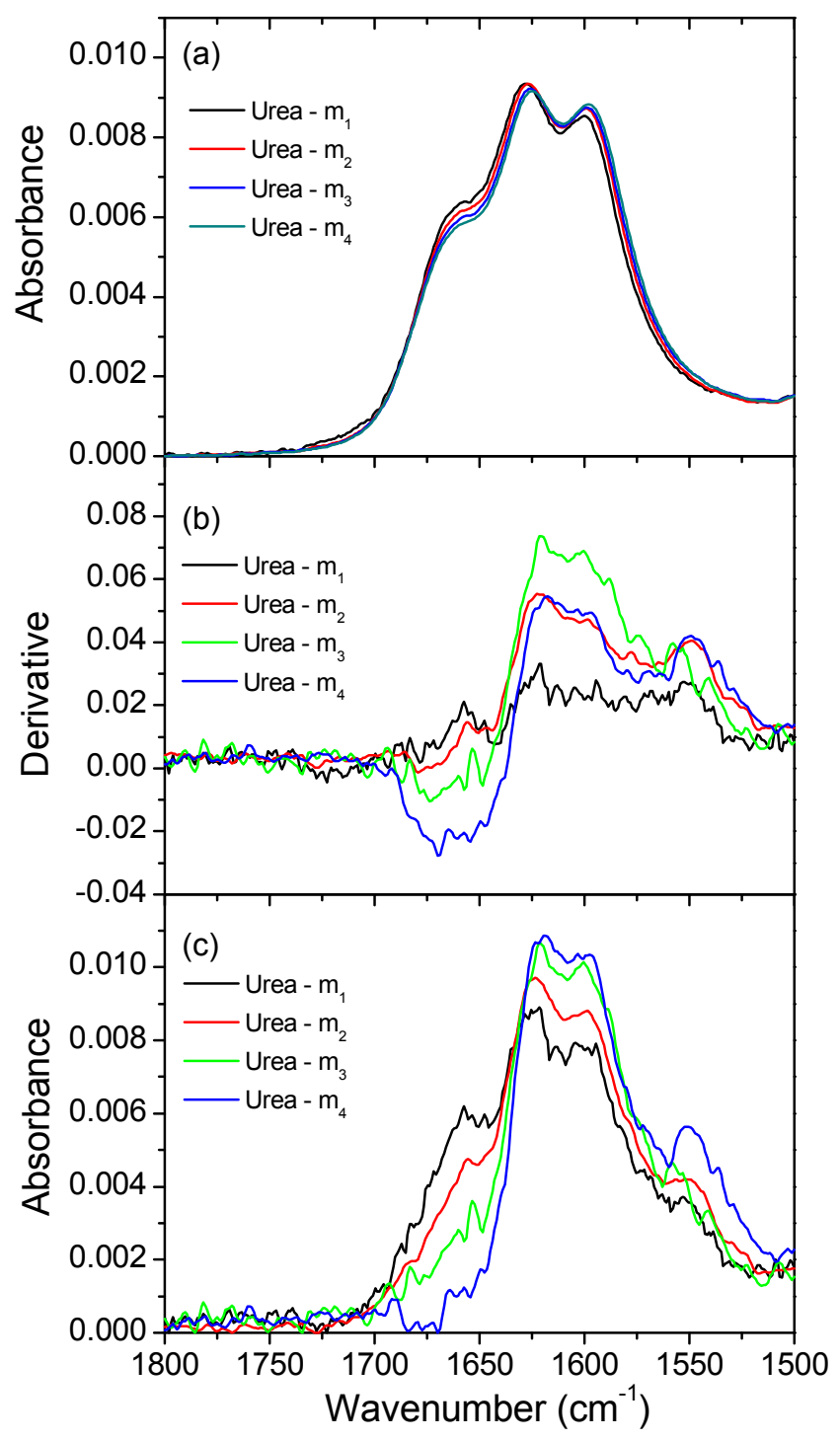

Figure S1. (a) Isolated spectra of urea in pure aqueous solutions corresponding to the $0^{\text {th }}$ row, $\mathrm{P}_{0}$, from Scheme $\mathrm{S} 1$, allow to calculate the solute spectrum extrapolated to $\mathrm{m}_{\mathrm{S}}=0 \mathrm{~mol} / \mathrm{kg}$ and the spectrum of solute affected by its concentration change (see Figures 1a-g). (b) Isolated spectra of urea affected by lysozyme and (c) derivatives $(d \varepsilon / d m)_{\mathrm{m} \rightarrow 0}$ at each wavenumber necessary to calculate them. 


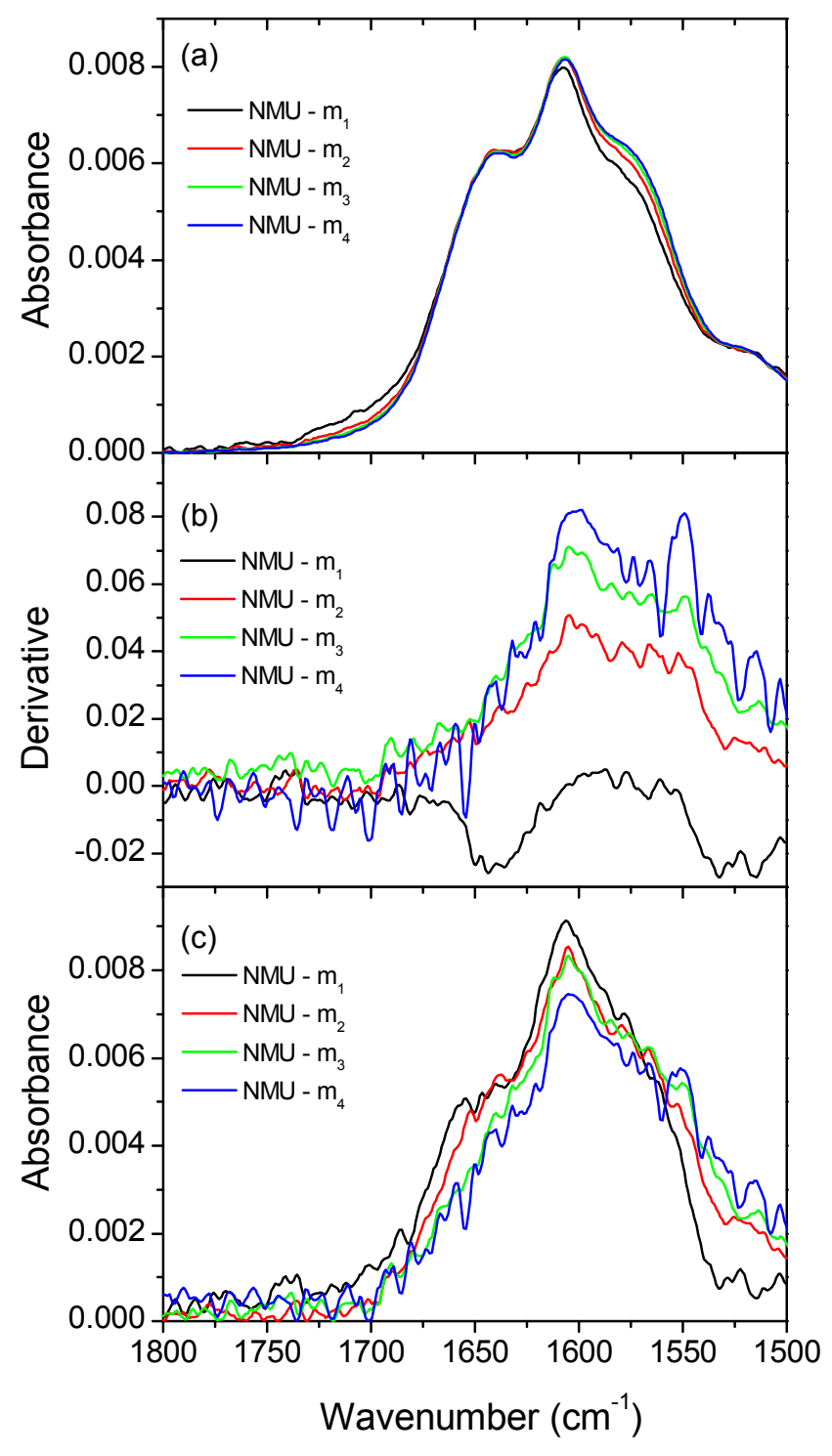

Figure S2. (a) Isolated spectra of NMU in pure aqueous solutions corresponding to the $0^{\text {th }}$ row, $\mathrm{P}_{0}$, from Scheme $\mathrm{S} 1$, allow to calculate the solute spectrum extrapolated to $\mathrm{m}_{\mathrm{S}}=0 \mathrm{~mol} / \mathrm{kg}$ and the spectrum of solute affected by its concentration change (see Figures 1a-g). (b) Isolated spectra of NMU affected by lysozyme and (c) derivatives $(d \varepsilon / d m)_{\mathrm{m} \rightarrow 0}$ at each wavenumber necessary to calculate them. 


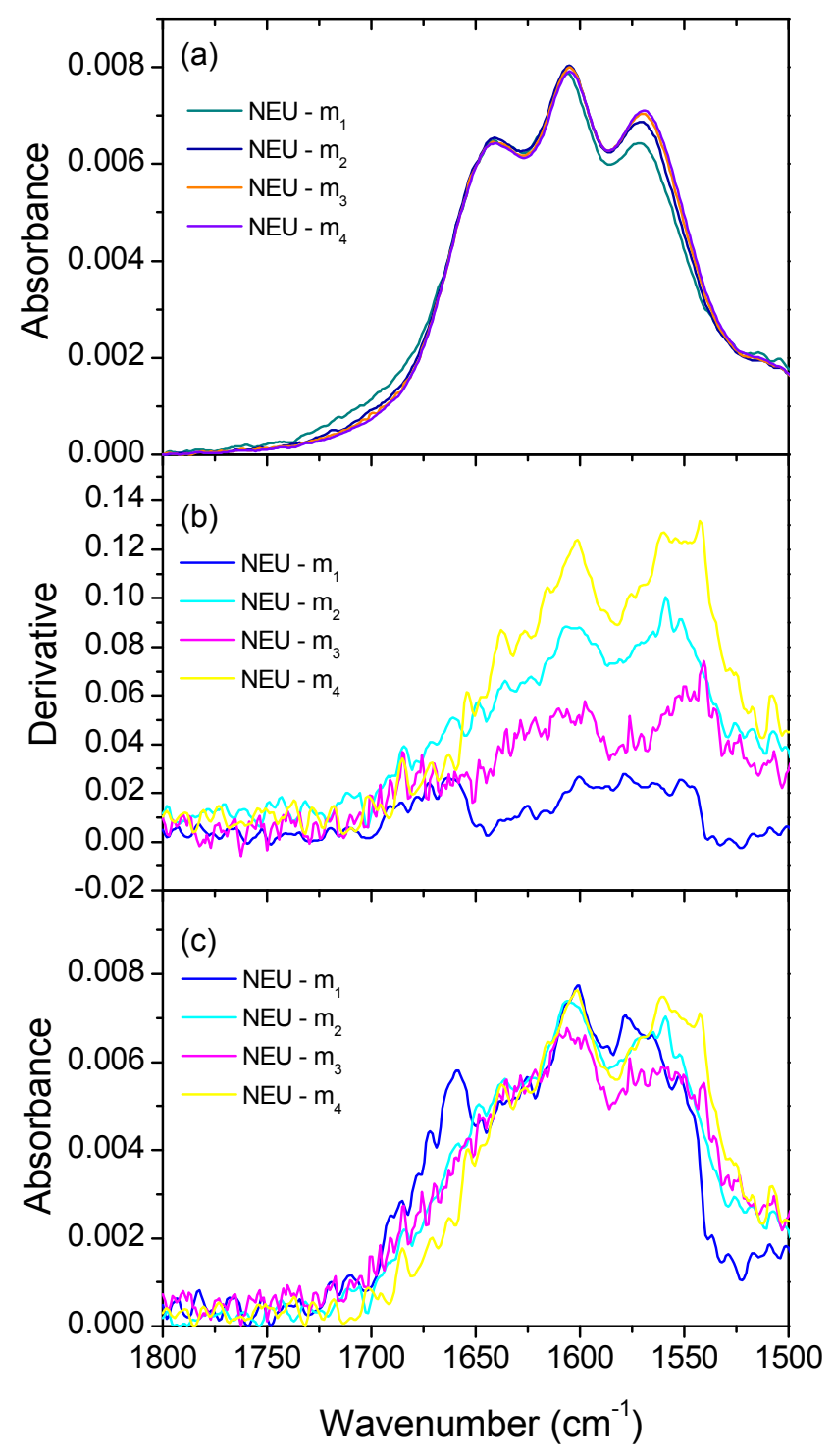

Figure S3. (a) Isolated spectra of NEU in pure aqueous solutions corresponding to the $0^{\text {th }}$ row, $\mathrm{P}_{0}$, from $\mathrm{Scheme} \mathrm{S} 1$, allow to calculate the solute spectrum extrapolated to $\mathrm{m}_{\mathrm{S}}=0 \mathrm{~mol} / \mathrm{kg}$ and the spectrum of solute affected by its concentration change (see Figures 1a-g). (b) Isolated spectra of NEU affected by lysozyme and (c) derivatives $(d \varepsilon / d m)_{\mathrm{m} \rightarrow 0}$ at each wavenumber necessary to calculate them. 


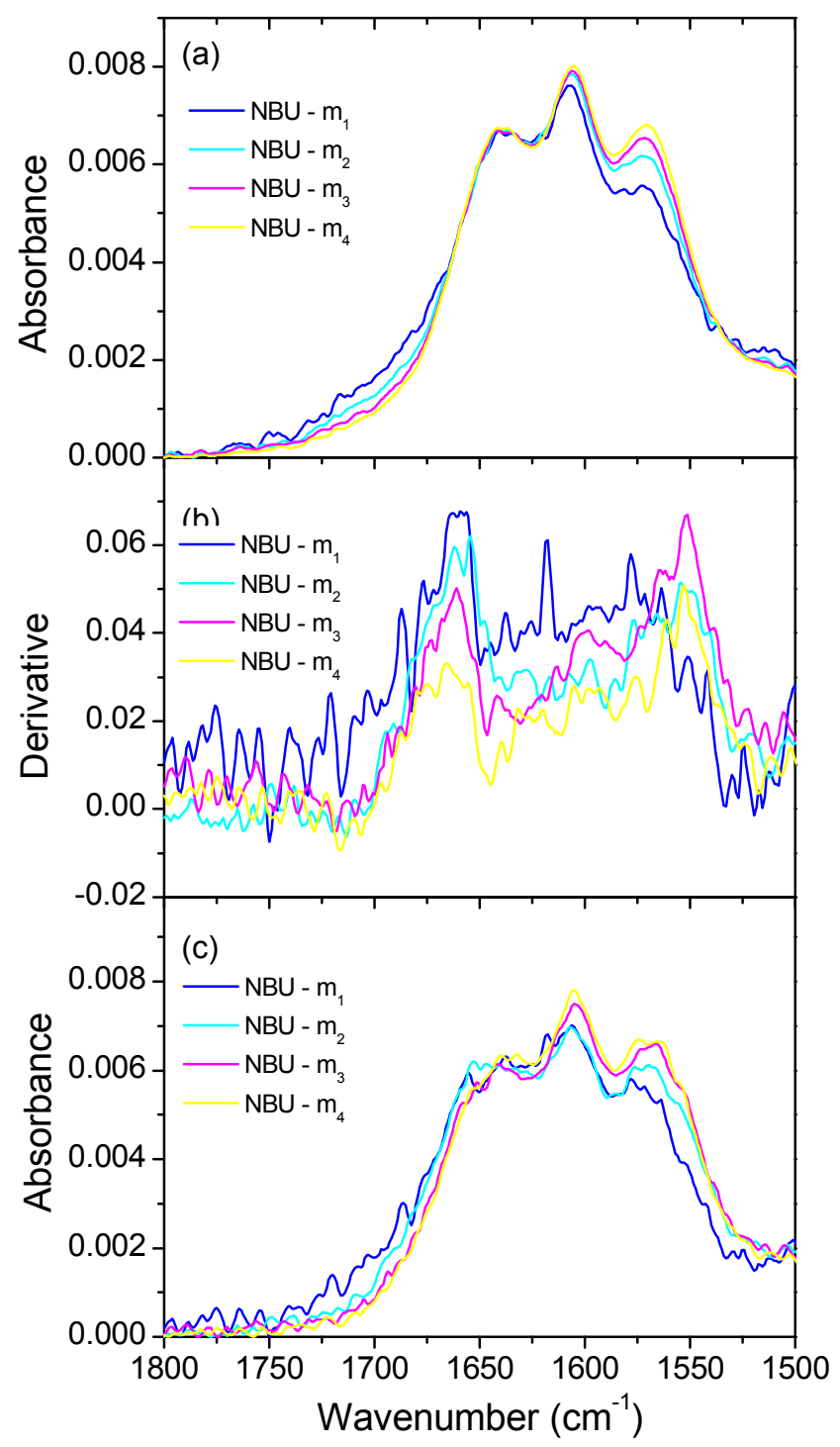

Figure S4. (a) Isolated spectra of NBU in pure aqueous solutions corresponding to the $0^{\text {th }}$ row, $\mathrm{P}_{0}$, from Scheme $\mathrm{S} 1$, allow to calculate the solute spectrum extrapolated to $\mathrm{m}_{\mathrm{S}}=0 \mathrm{~mol} / \mathrm{kg}$ and the spectrum of solute affected by its concentration change (see Figures 1a-g). (b) Isolated spectra of NBU affected by lysozyme and (c) derivatives $(d \varepsilon / d m)_{\mathrm{m} \rightarrow 0}$ at each wavenumber necessary to calculate them. 


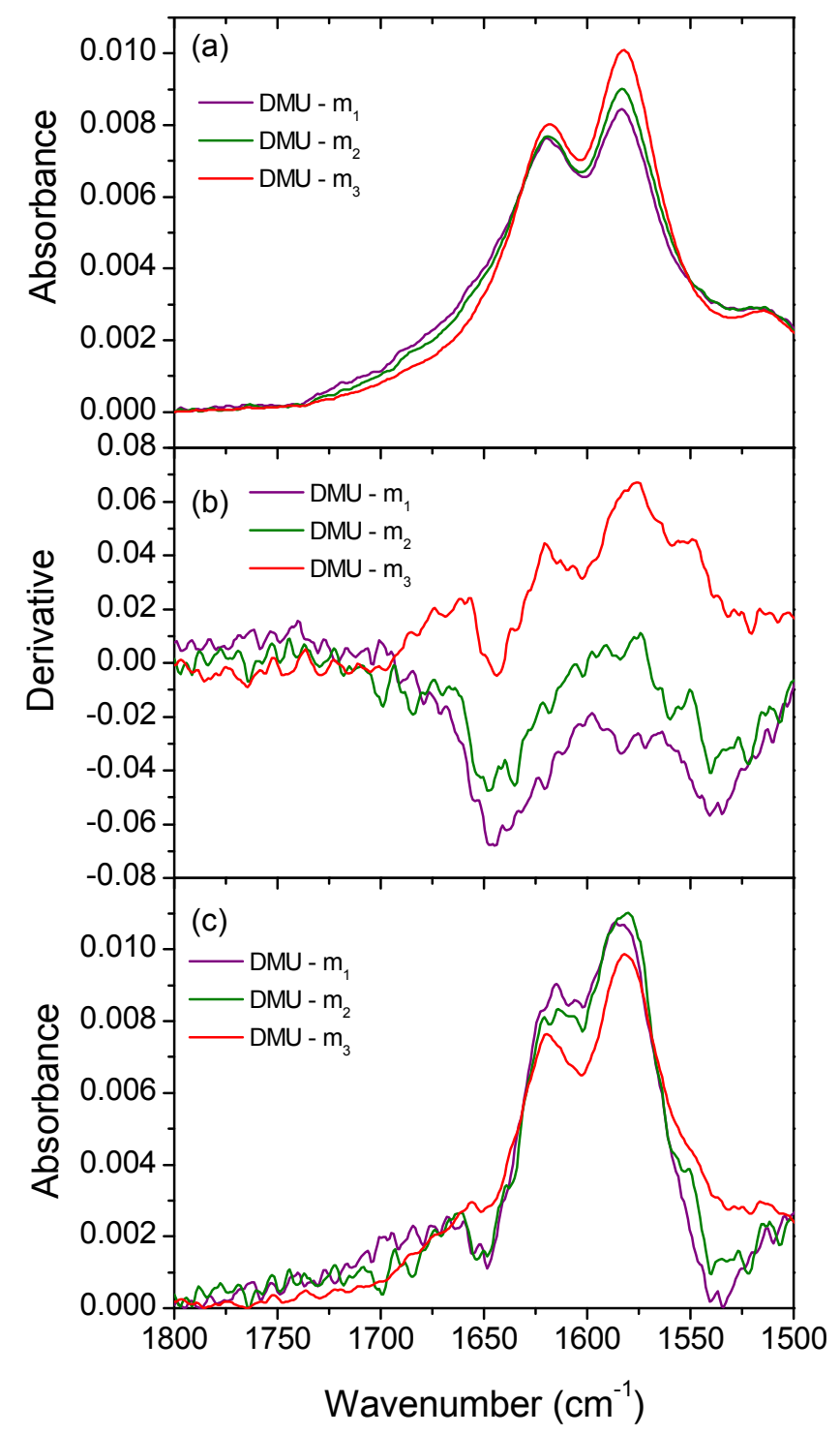

Figure S5. (a) Isolated spectra of DMU in pure aqueous solutions corresponding to the $0^{\text {th }}$ row, $\mathrm{P}_{0}$, from Scheme $\mathrm{S} 1$, allow to calculate the solute spectrum extrapolated to $\mathrm{m}_{\mathrm{S}}=0 \mathrm{~mol} / \mathrm{kg}$ and the spectrum of solute affected by its concentration change (see Figures 1a-g). (b) Isolated spectra of DMU affected by lysozyme and (c) derivatives $(d \varepsilon / d m)_{\mathrm{m} \rightarrow 0}$ at each wavenumber necessary to calculate them. 


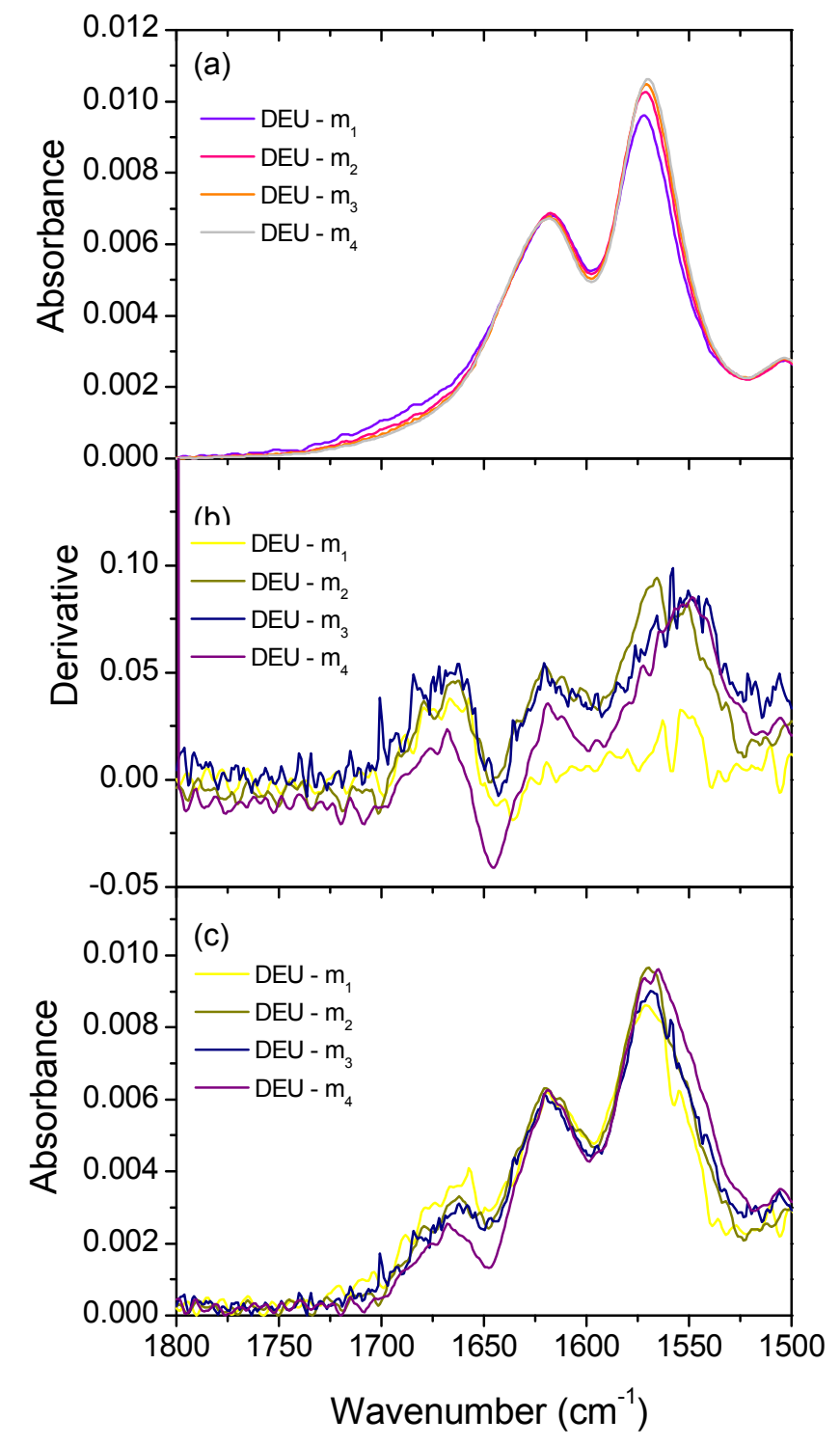

Figure S6. (a) Isolated spectra of DEU in pure aqueous solutions corresponding to the $0^{\text {th }}$ row, $\mathrm{P}_{0}$, from Scheme $\mathrm{S} 1$, allow to calculate the solute spectrum extrapolated to $\mathrm{m}_{\mathrm{S}}=0 \mathrm{~mol} / \mathrm{kg}$ and the spectrum of solute affected by its concentration change (see Figures 1a-g). (b) Isolated spectra of DEU affected by lysozyme and (c) derivatives $(d \varepsilon / d m)_{\mathrm{m} \rightarrow 0}$ at each wavenumber necessary to calculate them. 


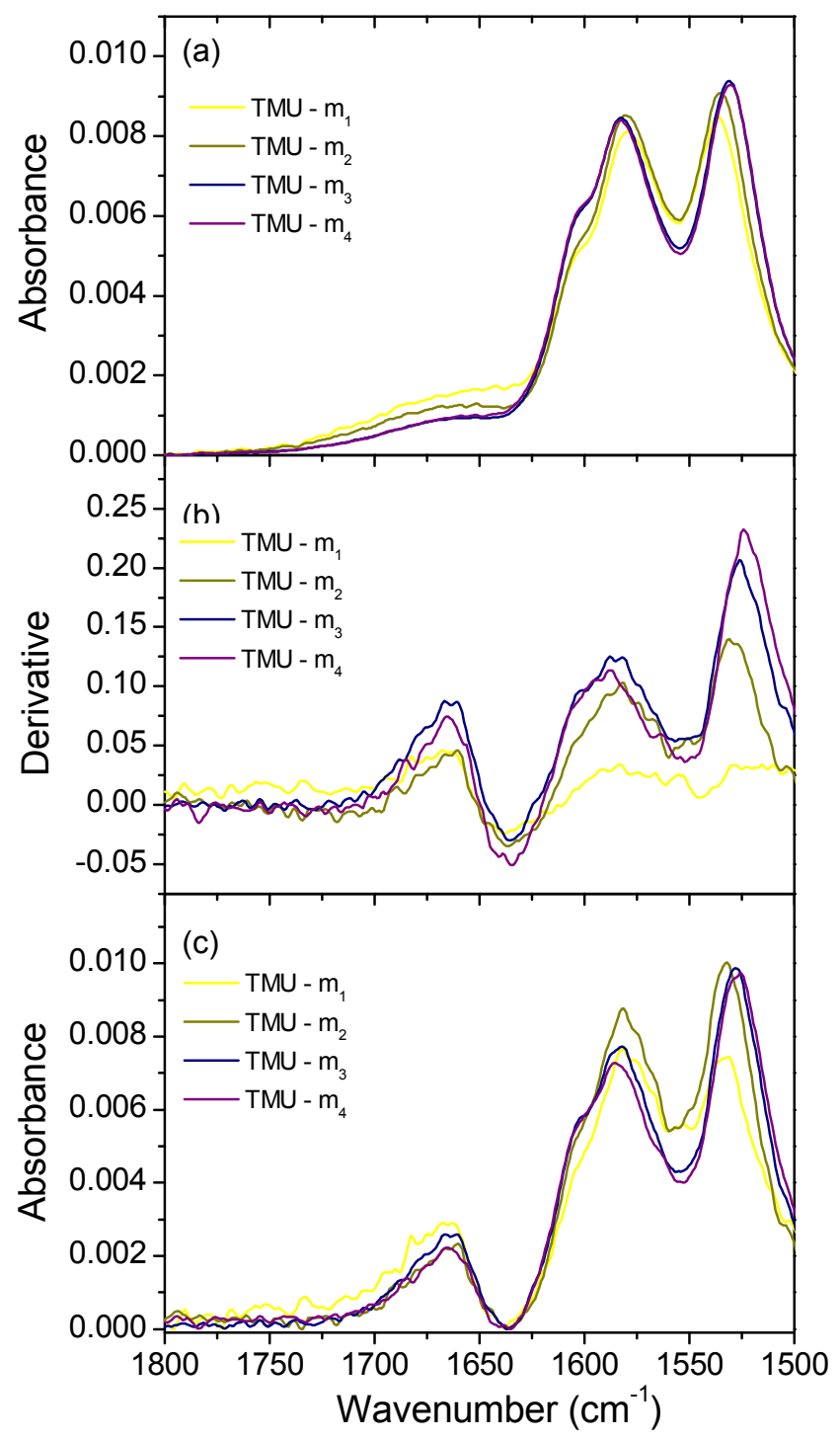

Figure S7. (a) Isolated spectra of TMU in pure aqueous solutions corresponding to the $0^{\text {th }}$ row, $\mathrm{P}_{0}$, from Scheme $\mathrm{S} 1$, allow to calculate the solute spectrum extrapolated to $\mathrm{m}_{\mathrm{S}}=0 \mathrm{~mol} / \mathrm{kg}$ and the spectrum of solute affected by its concentration change (see Figures 1a-g). (b) Isolated spectra of TMU affected by lysozyme and (c) derivatives $(d \varepsilon / d m)_{\mathrm{m} \rightarrow 0}$ at each wavenumber necessary to calculate them. 


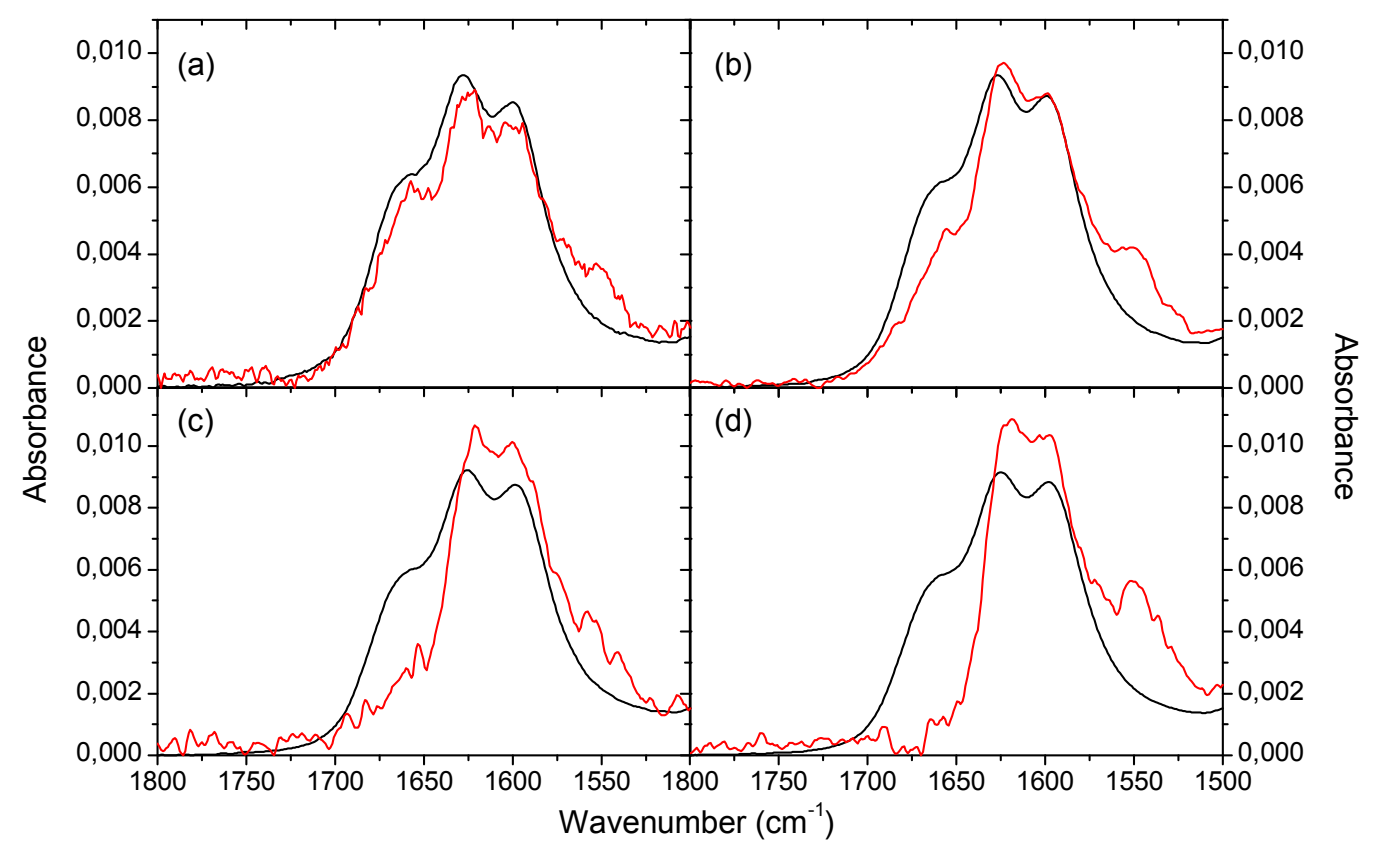

Figure S8. (a-d) Isolated spectra of Urea in pure aqueous solutions (black) and isolated spectra of urea affected by lysozyme (red); Figures (a-d) correspond to molalities $\mathrm{m}_{1}-\mathrm{m}_{4}$, respectively, according to Table S1. 


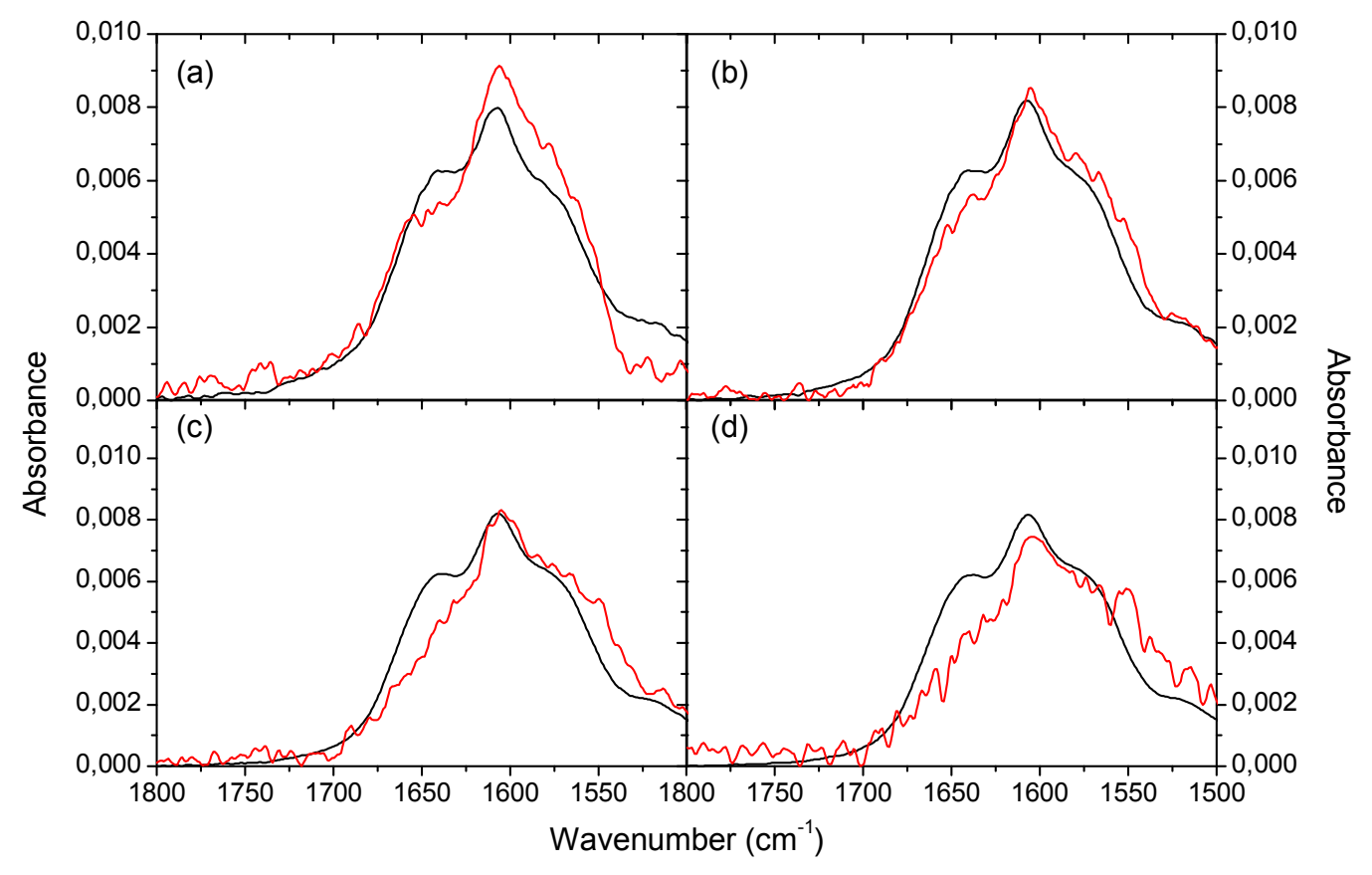

Figure S9. (a-d) Isolated spectra of NMU in pure aqueous solutions (black) and isolated spectra of NMU affected by lysozyme (red); Figures (a-d) correspond to molalities $\mathrm{m}_{1}-\mathrm{m}_{4}$, respectively, according to Table $\mathrm{S} 1$. 


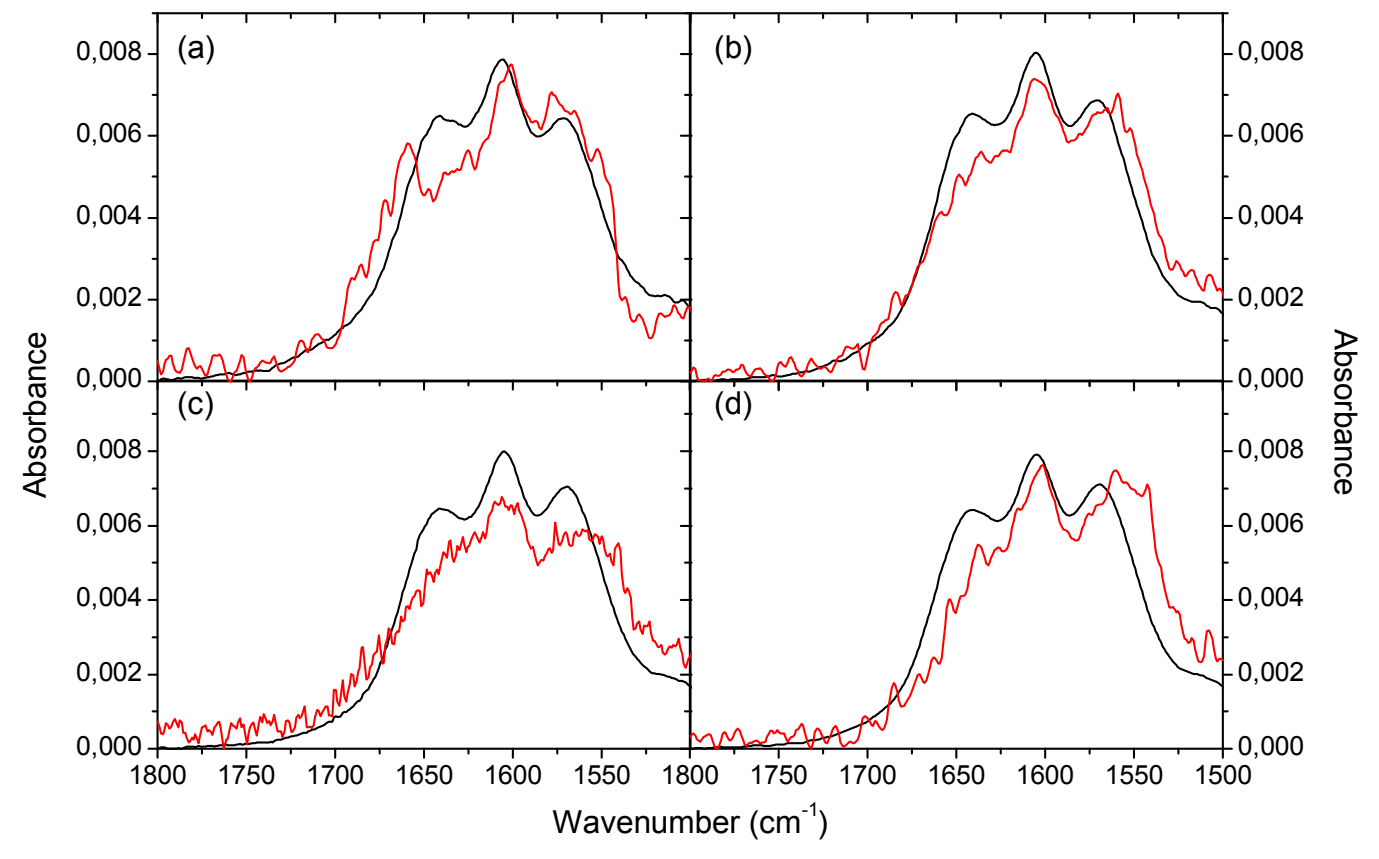

Figure S10. (a-d) Isolated spectra of NEU in pure aqueous solutions (black) and isolated spectra of NEU affected by lysozyme (red); Figures (a-d) correspond to molalities $\mathrm{m}_{1}-\mathrm{m}_{4}$, respectively, according to Table S1. 


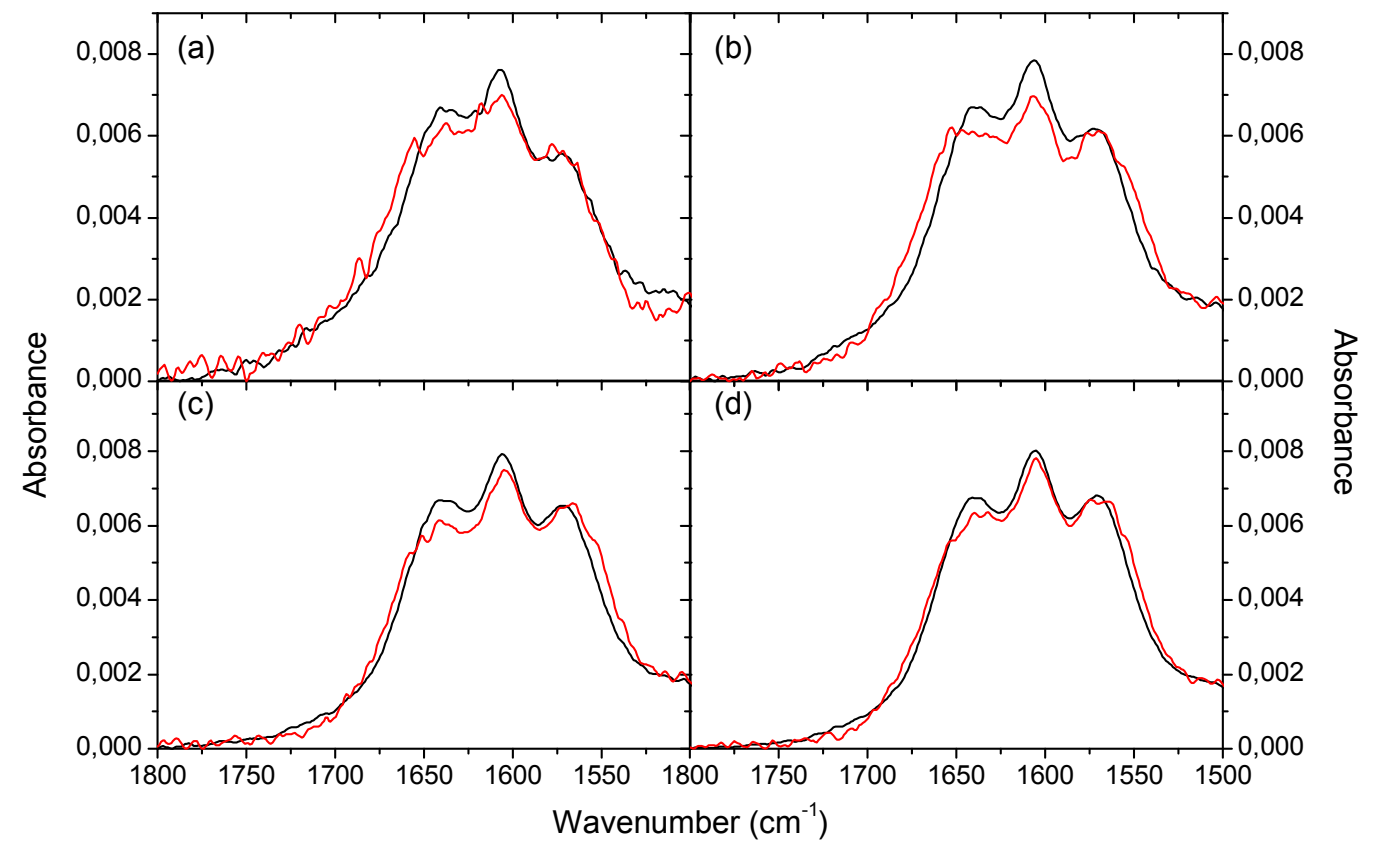

Figure S11. (a-d) Isolated spectra of NBU in pure aqueous solutions (black) and isolated spectra of NBU affected by lysozyme (red); Figures (a-d) correspond to molalities $\mathrm{m}_{1}-\mathrm{m}_{4}$, respectively, according to Table $\mathrm{S} 1$. 


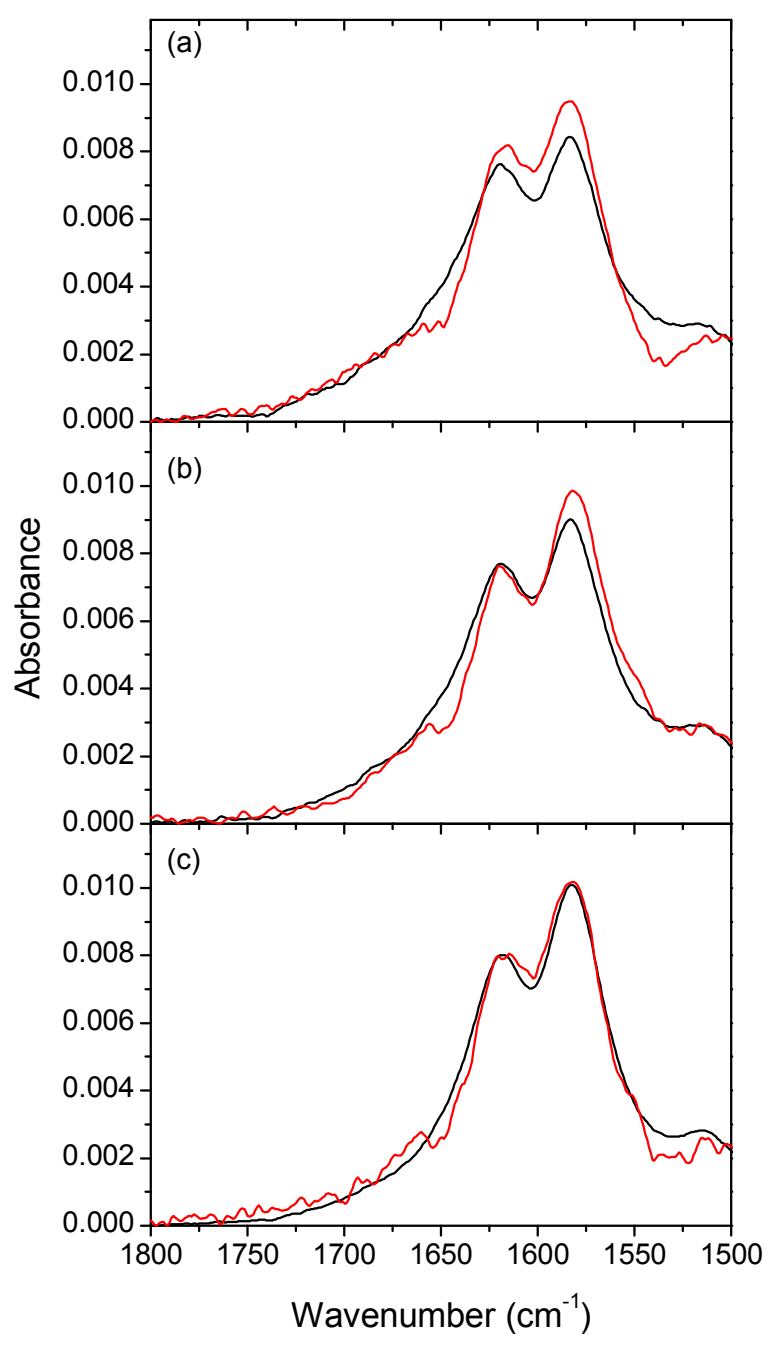

Figure S12. (a-c) Isolated spectra of DMU in pure aqueous solutions (black) and isolated spectra of DMU affected by lysozyme (red); Figures (a-c) correspond to molalities $\mathrm{m}_{1}-\mathrm{m}_{3}$, respectively, according to Table S1. 


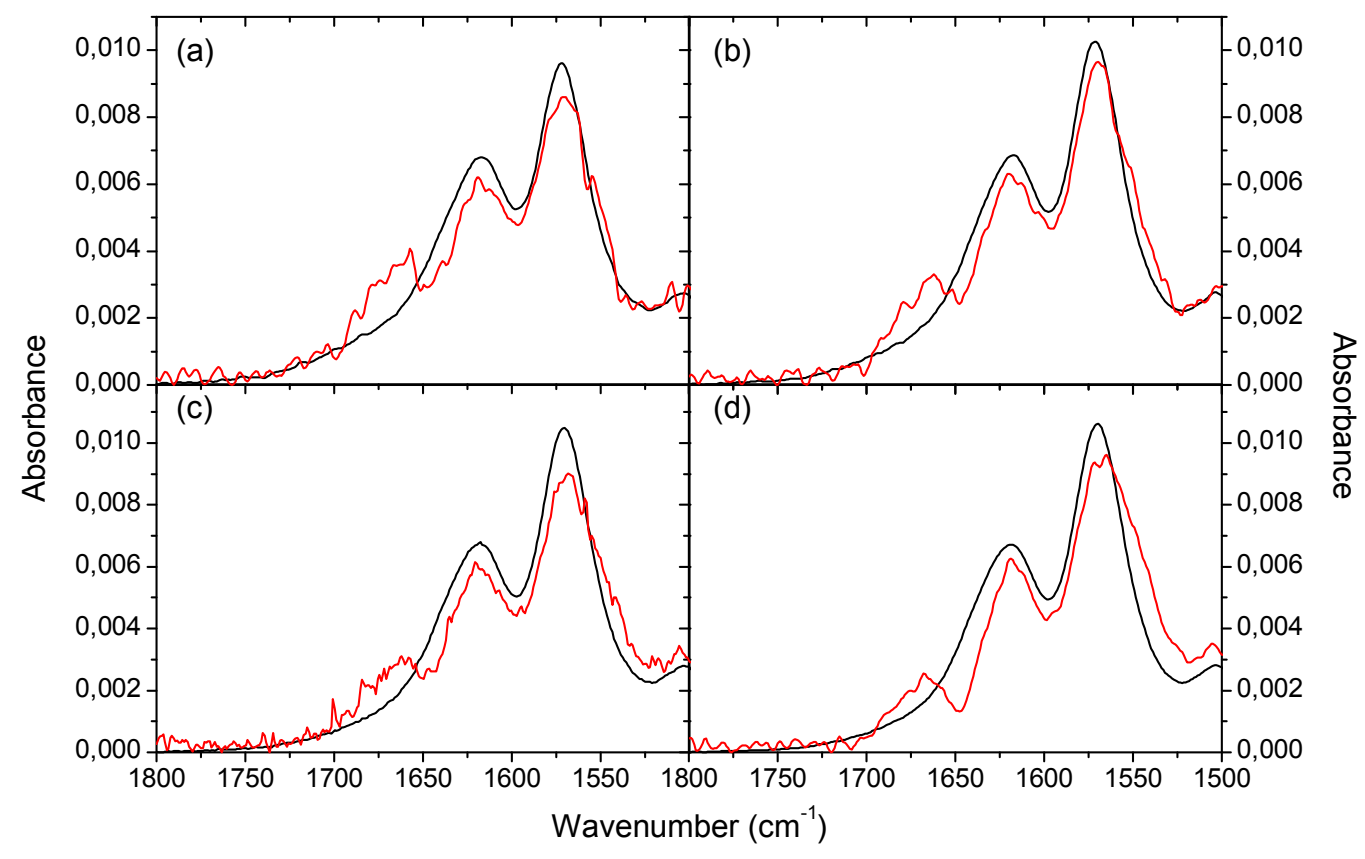

Figure S13. (a-d) Isolated spectra of DEM in pure aqueous solutions (black) and isolated spectra of DEM affected by lysozyme (red); Figures (a-d) correspond to molalities $\mathrm{m}_{1}-\mathrm{m}_{4}$, respectively, according to Table $\mathrm{S} 1$. 


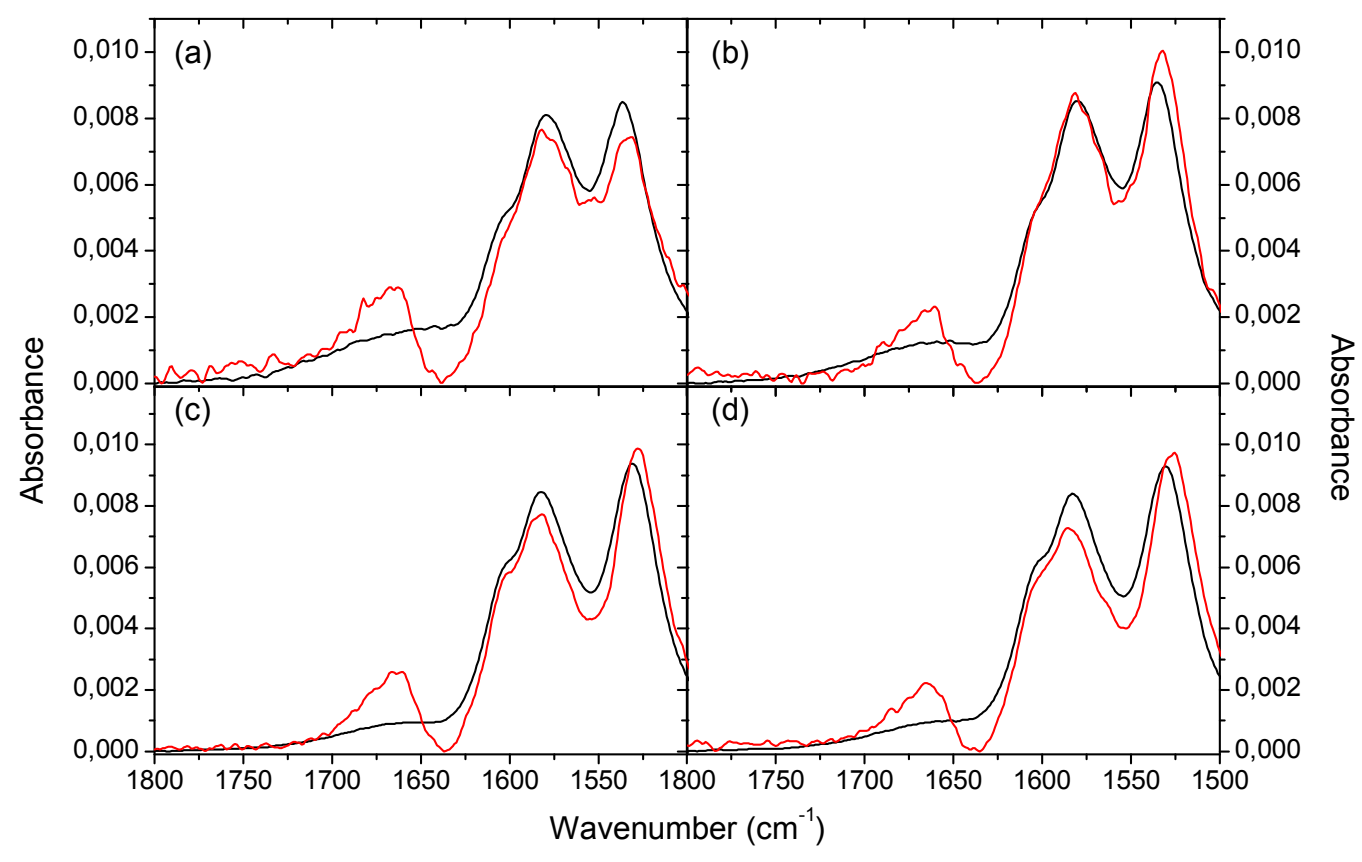

Figure S14. (a-d) Isolated spectra of TMU in pure aqueous solutions (black) and isolated spectra of TMU affected by lysozyme (red); Figures (a-d) correspond to molalities $\mathrm{m}_{1}-\mathrm{m}_{4}$, respectively, according to Table $\mathrm{S} 1$. 


\section{S2. DSC results}

Urea and all its derivatives lower the calorimetric enthalpy of denaturation, and NBU lowers it the most. This indicate that the denatured state is stabilized in regard to the native state of lysozyme. However in the presence of some urea derivatives an additional exothermic process occurs overlapping the DSC denaturation curve. Due to its high width and simple polynomial-like shape the process can be easily subtracted from the thermograms. It can be assigned to the decomposition of urea derivatives, probably catalyzed by the calorimeter platinum cells. The decomposition rate might be determined by the presence of $-\mathrm{NH}_{2}$ group, as the exothermic process occurs only in the case of urea and its monosubstituted derivatives (NMU, NEU, NBU). In the case of disubstituted derivatives (DMU, DEU) the process is not visible and does not obscure the denaturation peak on protein thermograms. 


\section{S2.1. Results of calorimetry data analysis - Tables}

Table S2. Calorimetric parameters of lysozyme denaturation process in the presence of urea and its derivatives.

\begin{tabular}{c|c|cccccccc}
\hline & $C^{a}$ & $\Delta H_{c a l}{ }^{b}$ & $\Delta H_{v H}{ }^{c}$ & $\Delta H_{v H} / \Delta H_{\text {cal }}{ }^{d}$ & $\Delta S_{\text {cal }}{ }^{e}$ & $\Delta C_{p}{ }^{f}$ & $T_{m}{ }^{g}$ & $\Delta H_{\text {rescn }}{ }^{h}$ & $R^{i}$ \\
\hline Lysozyme & 0.0000 & 570 & 457 & 0.80 & 1.64 & 5.1 & 74.9 & 52 & 9 \\
\hline \multirow{3}{*}{$\mathrm{U}$} & 0.4754 & 550 & 393 & 0.71 & 1.47 & 15 & 73.2 & - & - \\
& 0.9508 & 515 & 349 & 0.68 & 1.49 & 23 & 71.9 & - & - \\
& 1.4255 & 455 & 358 & 0.79 & 1.33 & 27 & 70.3 & - & - \\
\hline \multirow{4}{*}{ NMU } & 0.4815 & 457 & 352 & 0.77 & 1.33 & 8 & 72.2 & - & - \\
& 0.9630 & 486 & 398 & 0.82 & 1.41 & 28.2 & 70.1 & - & - \\
& 1.4445 & 433.3 & 491 & 1.13 & 1.27 & 19.5 & 66.8 & - & - \\
\hline \multirow{2}{*}{ DMU } & 0.4683 & 407 & 397 & 0.97 & 1.18 & 5.4 & 71.4 & 74 & 18 \\
& 0.9366 & 425 & 430 & 1.01 & 1.25 & 5.7 & 68.3 & 100 & 24 \\
& 1.4049 & 387 & 455 & 1.18 & 1.14 & 5.2 & 65.3 & 108 & 28 \\
\hline \multirow{5}{*}{ NEU } & 0.5090 & 458 & 430 & 0.94 & 1.29 & 26.5 & 70.6 & - & - \\
& 1.0181 & 351 & 477 & 1.36 & 1.03 & 26.5 & 66.7 & - & - \\
& 1.5271 & 323 & 563 & 1.74 & 0.96 & 14.1 & 63.4 & - & - \\
\hline \multirow{3}{*}{ DEU } & 0.5023 & 350 & 402 & 1.15 & 1.03 & 4.6 & 67.3 & 39 & 11 \\
& 1.0046 & 360 & 365 & 1.01 & 1.08 & 2.8 & 61.1 & 80 & 22 \\
& 1.5069 & 340 & 440 & 1.29 & 1.03 & 3.2 & 55.4 & 58 & 17 \\
\hline \multirow{2}{*}{ NBU } & 0.1645 & 366 & 382 & 1.04 & 1.06 & 14.3 & 70 & - & - \\
& 0.3289 & 273 & 422 & 1.55 & 0.81 & 8.5 & 64.9 & - & - \\
& 0.4934 & 178 & 436 & 2.45 & 0.54 & 10.0 & 60.9 & - & - \\
\hline
\end{tabular}

${ }^{a}$ Osmolite concentration $\left(\mathrm{mol} \cdot \mathrm{dm}^{-3}\right) .{ }^{b}$ Calorimetric denaturation enthalpy $\left(\mathrm{kJ} \cdot \mathrm{mol}^{-1}\right) .{ }^{c}$ Van ' $\mathrm{t}$ Hoff's enthalpy $\left(\mathrm{kJ} \cdot \mathrm{mol}^{-1}\right) .{ }^{d}$ The van 't Hoff to calorimetric enthalpy ratio $\left(\mathrm{kJ} \cdot \mathrm{mol}^{-1}\right) .{ }^{e}$ Calorimetric entropy $\left(\mathrm{kJ} \cdot \mathrm{mol}^{-1} \cdot \mathrm{K}^{-1}\right) .{ }^{f}$ The change of heat excess capacity at $T_{m}\left(\mathrm{~kJ} \cdot \mathrm{mol}^{-1} \cdot \mathrm{K}^{-1}\right)$.

${ }^{g}$ Denaturation temperature (denaturation peak maximum) $\left({ }^{o} \mathrm{C}\right) \cdot{ }^{h}$ Calorimetric enthalpy of the protein in re-scan $\left(\mathrm{kJ} \cdot \mathrm{mol}^{-1}\right) .{ }^{i}$ Denaturation reversibility $\left(\Delta H_{\text {cal }} / \Delta H_{\text {rescn }}\right.$ ratio $)(\%)$. 
Table S3. Fitting parameters of two-state equilibrium model and Lumry-Eyring's irreversible model.

\begin{tabular}{c|c|ccc|cccccc}
\hline & & \multicolumn{7}{|c|}{ Two-state equilibrium } & \multicolumn{6}{c}{ Lumry'ego- Eyring's irreversible model } \\
\hline & $C^{a}$ & $\Delta H_{N-D}{ }^{b}$ & $T_{m, N-D}{ }^{c}$ & $\Delta S_{N-D}{ }^{d}$ & $\Delta H_{L-E}{ }^{e}$ & $T_{m, L-E}{ }^{f}$ & $\Delta S_{L-E}{ }^{g}$ & $E_{a}{ }^{h}$ & $T^{*}{ }^{\prime}$ \\
& & & & & & & & & \\
\hline Lysozyme & 0.0000 & 530 & 74.8 & 1.52 & 526 & 74.8 & 1.51 & 568 & 83.4 \\
\hline \multirow{3}{*}{$\mathrm{U}$} & 0.4754 & 469 & 72.9 & 1.36 & 462 & 72.8 & 1.34 & 489 & 80.7 \\
& 0.9508 & 470 & 71.3 & 1.36 & 481 & 71.2 & 1.40 & 89 & $>1000$ \\
& 1.4255 & 458 & 70.1 & 1.33 & 440 & 71.0 & 1.28 & 4 & $>1000$ \\
\hline \multirow{3}{*}{ NMU } & 0.4815 & 432 & 71.8 & 1.25 & 412 & 72.6 & 1.19 & 5 & $>1000$ \\
& 0.9630 & 454 & 69.8 & 1.32 & 405 & 71.3 & 1.18 & 3 & $>1000$ \\
& 1.4445 & 457 & 66.3 & 1.35 & 448 & 67.1 & 1.32 & 5 & $>1000$ \\
\hline \multirow{2}{*}{ DMU } & 0.4683 & 432 & 71.4 & 1.25 & 403 & 73.0 & 1.16 & 15 & 171.5 \\
& 0.9366 & 441 & 68.1 & 1.29 & 418 & 69.4 & 1.22 & 5 & $>1000$ \\
& 1.4049 & 425 & 65.3 & 1.26 & 392 & 66.7 & 1.15 & 60 & 86.3 \\
\hline \multirow{3}{*}{ NEU } & 0.5090 & 447 & 70.2 & 1.30 & 414 & 71.7 & 1.20 & 4 & $>1000$ \\
& 1.0181 & 403 & 66.3 & 1.19 & 330 & 68.9 & 0.96 & 96 & 72.6 \\
& 1.5271 & 408 & 63.3 & 1.21 & 344 & 65.7 & 1.02 & 100 & 68.9 \\
\hline \multirow{2}{*}{ DEU } & 0.5023 & 391 & 67.1 & 1.15 & 351 & 69.5 & 1.02 & 48 & 83.6 \\
& 1.0046 & 392 & 60.8 & 1.17 & 358 & 62.5 & 1.07 & 49 & 82.9 \\
& 1.5069 & 384 & 55.3 & 1.17 & 349 & 56.9 & 1.06 & 60 & 72.8 \\
\hline \multirow{2}{*}{ NBU } & 0.1645 & 380 & 69.3 & 1.11 & - & - & - & - & - \\
& 0.3289 & 324 & 64.3 & 0.96 & - & - & - & - & - \\
& 0.4934 & - & - & - & - & - & - & - & - \\
\hline
\end{tabular}

${ }^{a}$ Osmolite concentration $\left(\mathrm{mol} \cdot \mathrm{dm}^{-3}\right) .{ }^{b}$ Denaturation enthalpy (from equilibrium model) $\left(\mathrm{kJ} \cdot \mathrm{mol}^{-1}\right) .{ }^{c}$ Denaturation temperature (from equilibrium model) $\left({ }^{o} \mathrm{C}\right) .{ }^{d}$ Denaturation entropy (from equilibrium model) $\left(\mathrm{kJ} \cdot \mathrm{mol}^{-1} \cdot \mathrm{K}^{-1}\right){ }^{e}$ Denaturation enthalpy (from Lumry-Eyring's model) $\left(\mathrm{kJ} \cdot \mathrm{mol}^{-1}\right) .{ }^{f}$ Denaturation temperature (from Lumry-Eyring's model) $\left({ }^{o} \mathrm{C}\right) .{ }^{g}$ Denaturation entropy (from Lumry-Eyring's model) $\left(\mathrm{kJ} \cdot \mathrm{mol}^{-1} \cdot \mathrm{K}^{-1}\right) .{ }^{h}$ Activation energy (from Lumry-Eyring's model) $\left(\mathrm{kJ} \cdot \mathrm{mol}^{-1}\right) .{ }^{i}$ Temperature where $k=1 \mathrm{~min}^{-1}$ (from Lumry-Eyring's model) $\left({ }^{\circ} \mathrm{C}\right)$. 
Table S4. Calorimetric parameters of lysozyme denaturation process in the presence of N-nbuthylurea. Fitting parameters of the two-state kinetic irreversible model.

\begin{tabular}{ccccccc}
\hline$C^{a}$ & $\Delta H^{b}$ & $T_{\max }{ }^{c}$ & $E_{a}{ }^{d}$ & $T^{* e}$ & $k^{(25) f}$ & $t_{1 / 2}^{(25)}{ }^{g}$ \\
\hline 0.1645 & 366 & 70.0 & 267 & 75.0 & $1.91 \cdot 10^{-7}$ & 7 \\
0.3289 & 279 & 64.9 & 267 & 69.8 & $7.58 \cdot 10^{-7}$ & 2 \\
0.4934 & 184 & 60.9 & 282 & 65.4 & $1.23 \cdot 10^{-6}$ & 1 \\
\hline
\end{tabular}

${ }^{a}$ Osmolite concentration $\left(\mathrm{mol} \cdot \mathrm{dm}^{-3}\right) .{ }^{b}$ Denaturation enthalpy $\left(\mathrm{kJ} \cdot \mathrm{mol}^{-1}\right) .{ }^{c}$ Denaturation temperature $\left({ }^{o} \mathrm{C}\right) .{ }^{d}$ Activation energy $\left(\mathrm{kJ} \cdot \mathrm{mol}^{-1}\right) .{ }^{e}$ Temperature where $k=1 \mathrm{~min}^{-1}\left({ }^{o} \mathrm{C}\right) .{ }^{f}$ Rate constant at $25{ }^{\circ} \mathrm{C}\left(\mathrm{min}^{-1}\right) .{ }^{g}$ Half-life at $25^{\circ} \mathrm{C}$ (Years). 


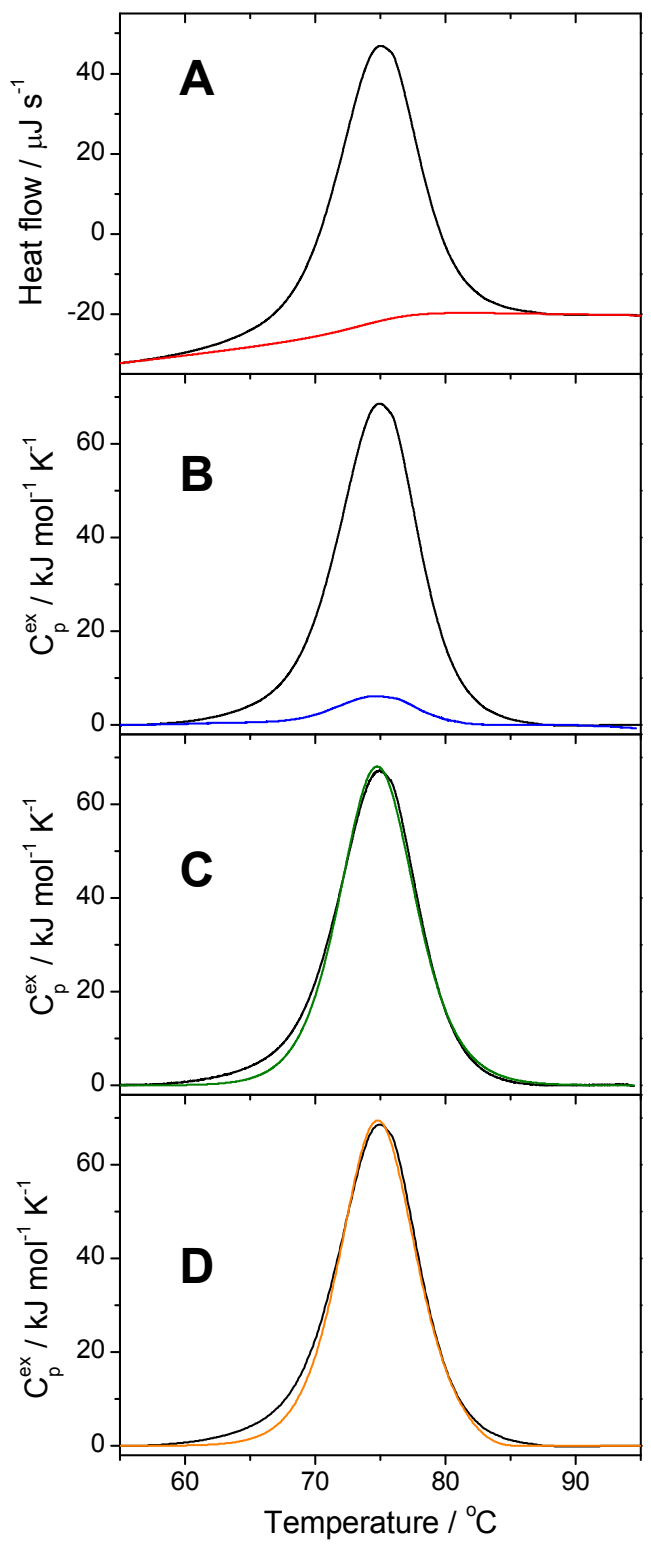

Figure S15. (A) Thermogram of lysozyme (in phosphate buffer, black line) and baseline (red line). (B) baseline-corrected thermogram from Figure A (black line) and re-scan (blue line). (C) The result of the two-state reversible model fitting (green line) to the baseline-corrected thermogram (black line). (D) The result of the Lumry-Eyring's irreversible model fitting (orange line) to the baseline-corrected thermogram (black line). 


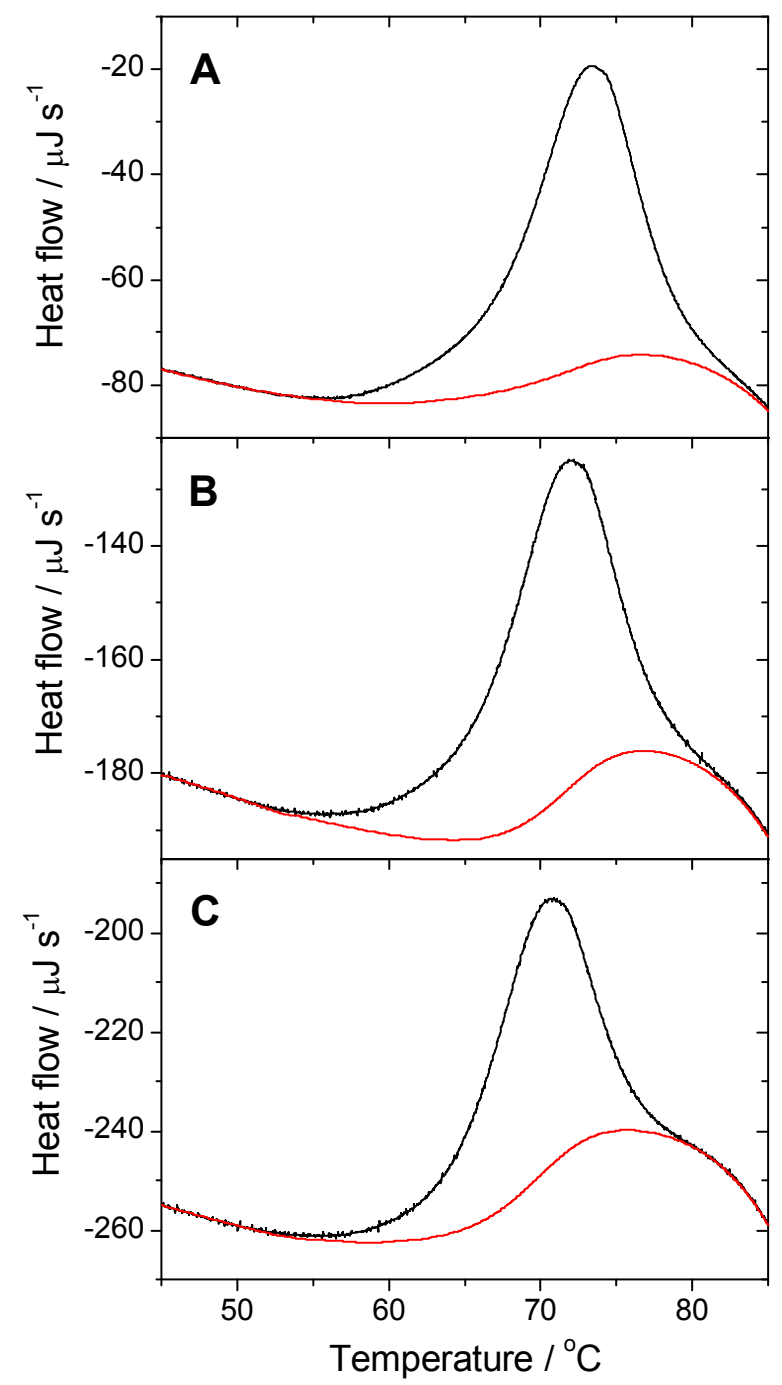

Figure S16. (A,B,C) Thermograms of lysozyme in the presence of urea at $0.4754,0.9508$ and $1.4255 \mathrm{~mol} \cdot \mathrm{dm}^{-3}$ concentrations, respectively (black lines). Baselines are marked with red lines. 


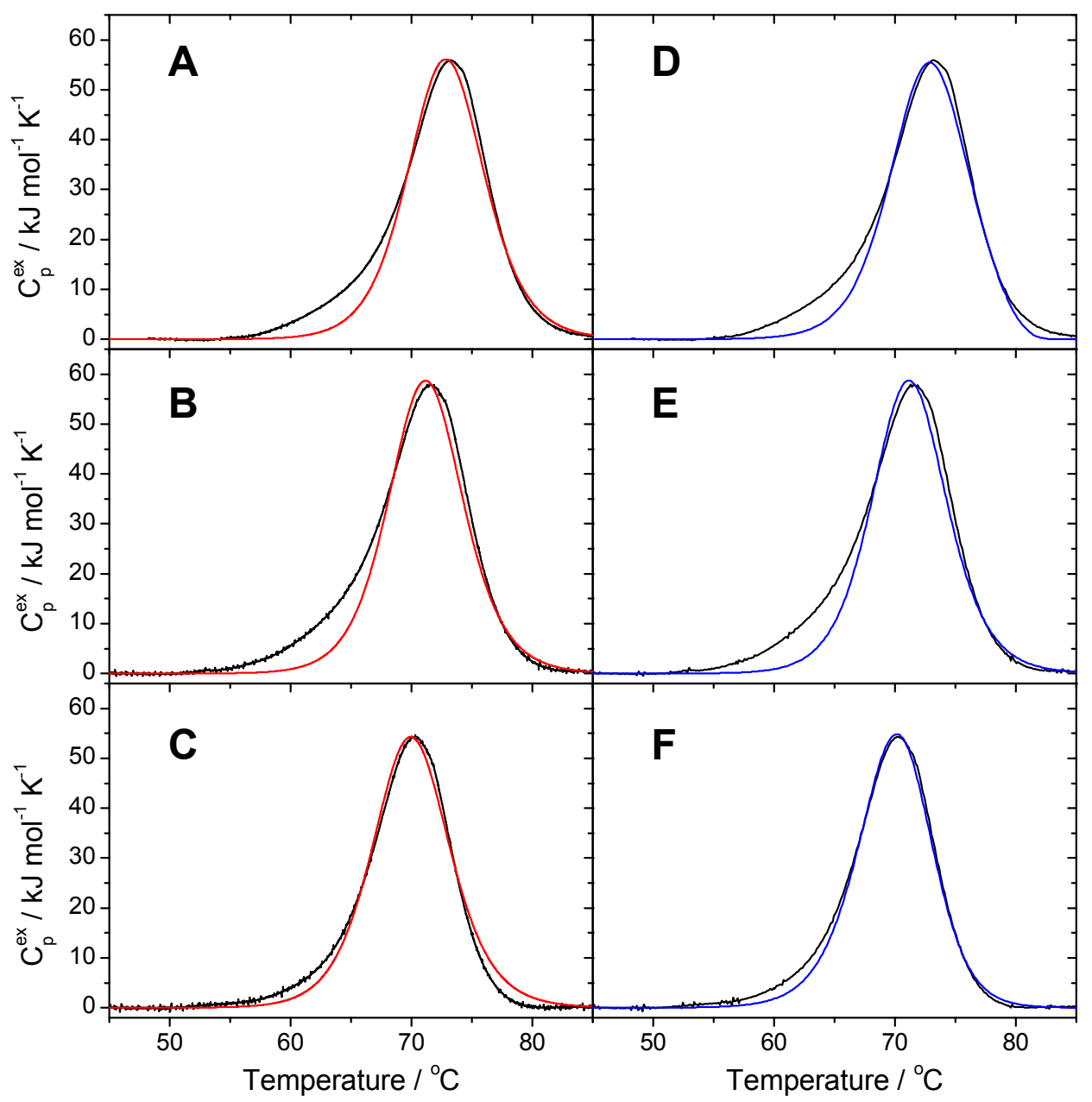

Figure S17. (A,B,C) Results of the two-state reversible model fitting (red lines) to the baseline-corrected thermograms (black lines) corresponding to $0.4754,0.9508$ and 1.4255 $\mathrm{mol} \cdot \mathrm{dm}^{-3}$ urea, respectively. (D) Results of the Lumry-Eyring's irreversible model fitting (blue lines) to the same baseline-corrected thermograms (black lines). 


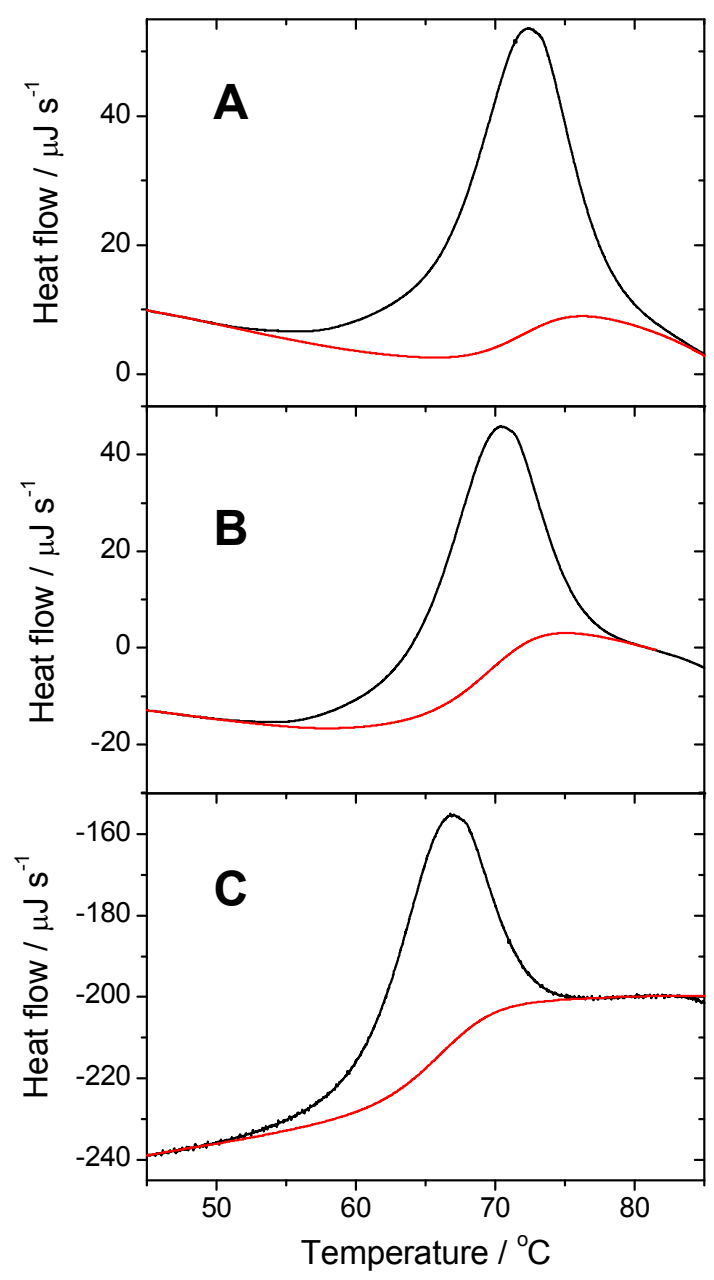

Figure S18. (A,B,C) Thermograms of lysozyme in the presence of NMU at $0.4815,0.9630 \mathrm{i}$ $1.4445 \mathrm{~mol} \cdot \mathrm{dm}^{-3}$ concentrations, respectively (black lines). Baselines are marked with red lines. 


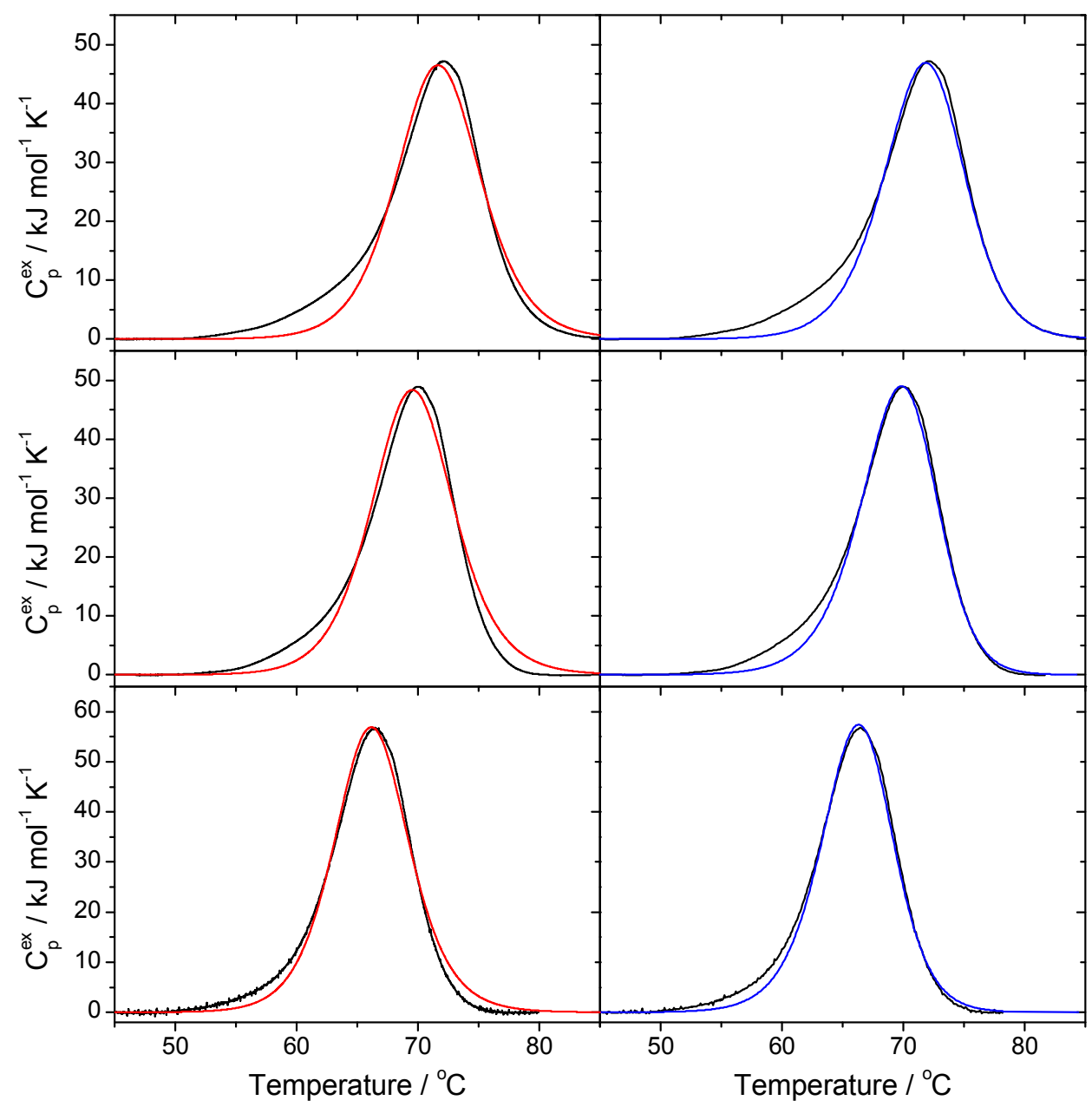

Figure S19. (A,B,C) Results of the two-state reversible model fitting (red lines) to the baseline-corrected thermograms (black lines) corresponding to $0.4815,0.9630 \mathrm{i} 1.4445$ $\mathrm{mol} \cdot \mathrm{dm}^{-3}$ NMU solutions, respectively. (D) Results of the Lumry-Eyring's irreversible model fitting (blue lines) to the same baseline-corrected thermograms (black lines). 


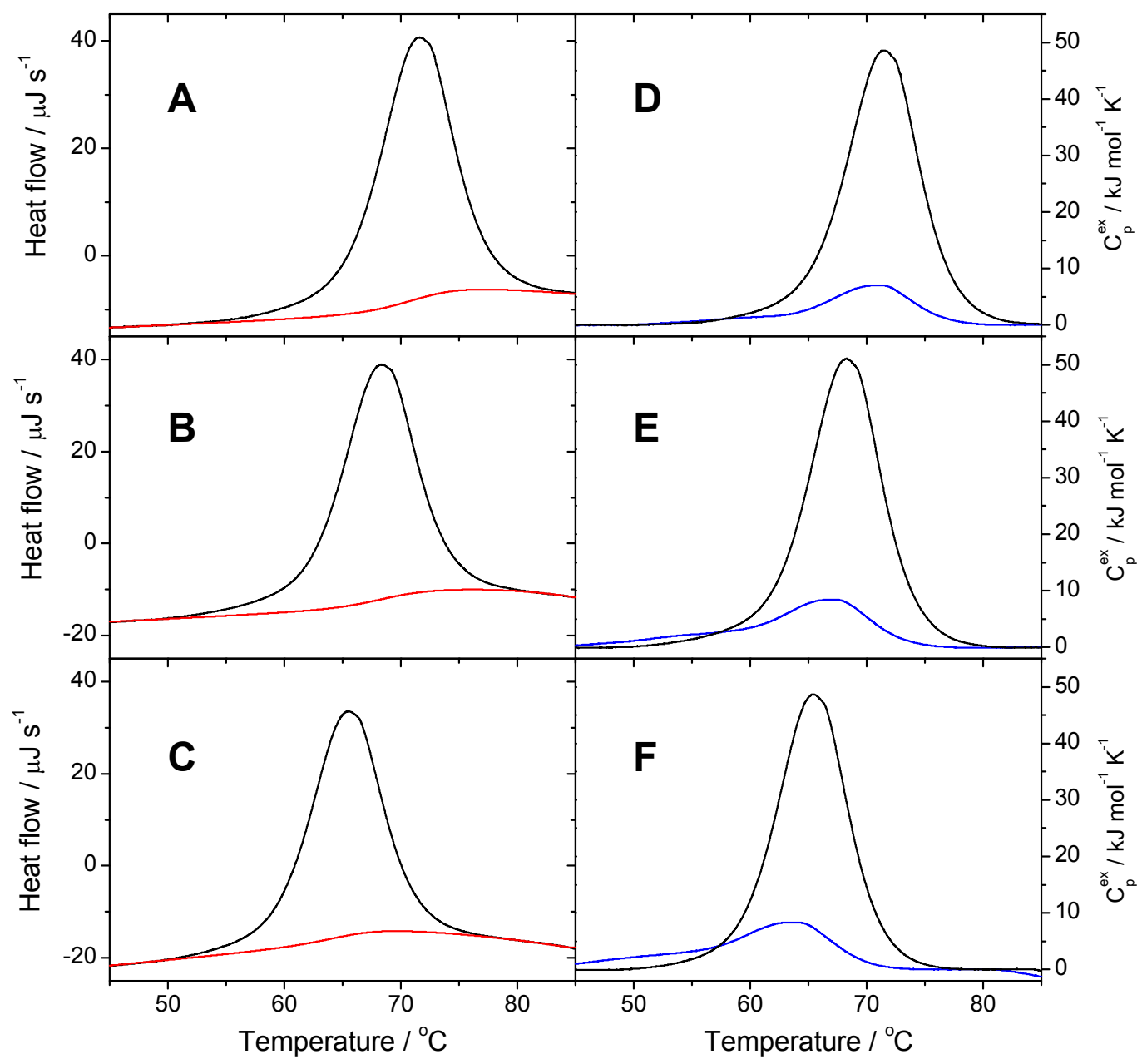

Figure S20. (A,B,C) Thermograms of lysozyme in the presence of DMU at $0.4683,0.9366$ and $1,4049 \mathrm{~mol} \cdot \mathrm{dm}^{-3}$ concentrations, respectively (black lines). Baselines are marked with red lines. (D,E,F) Baseline-corrected thermograms from Figures A,B, and C (black lines) and rescans (blue lines). 


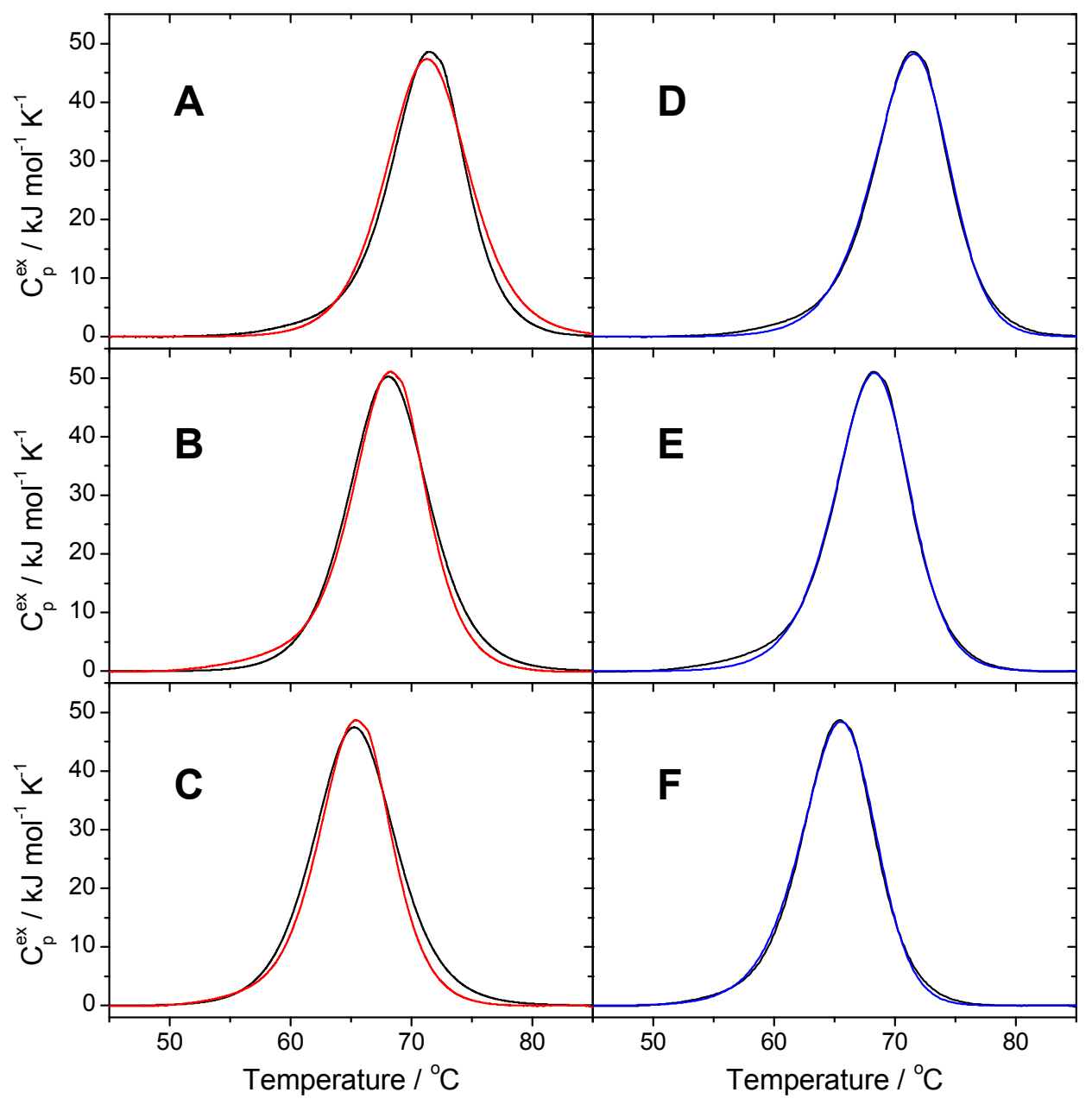

Figure S21. (A,B,C) Results of the two-state reversible model fitting (red lines) to the baseline-corrected thermograms (black lines) corresponding to $0.4683,0.9366$ and 1,4049 $\mathrm{mol} \cdot \mathrm{dm}^{-3}$ DMU solutions, respectively. (D) Results of the Lumry-Eyring's irreversible model fitting (blue lines) to the same baseline-corrected thermograms (black lines). 


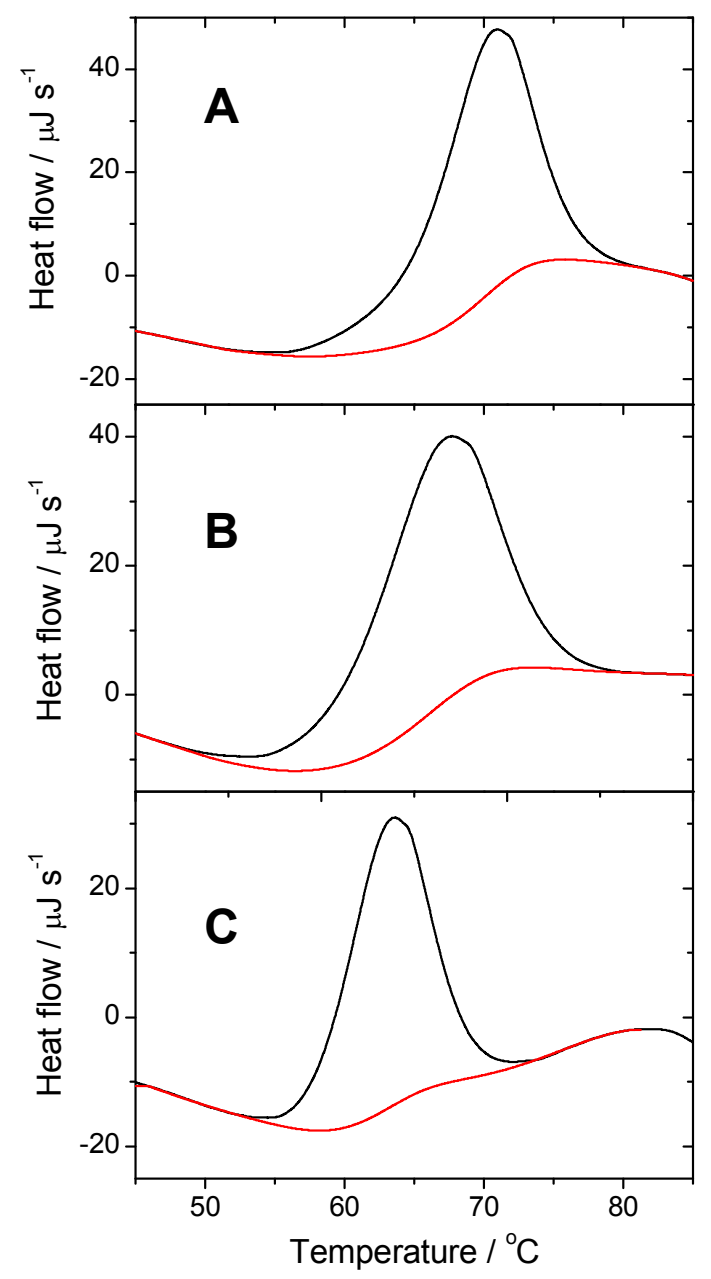

Figure S22. (A,B,C) Thermograms of lysozyme in the presence of NEU at 0.5090, 1.0181 and $1.5271 \mathrm{~mol} \cdot \mathrm{dm}^{-3}$ concentrations, respectively (black lines). Baselines are marked with red lines. 


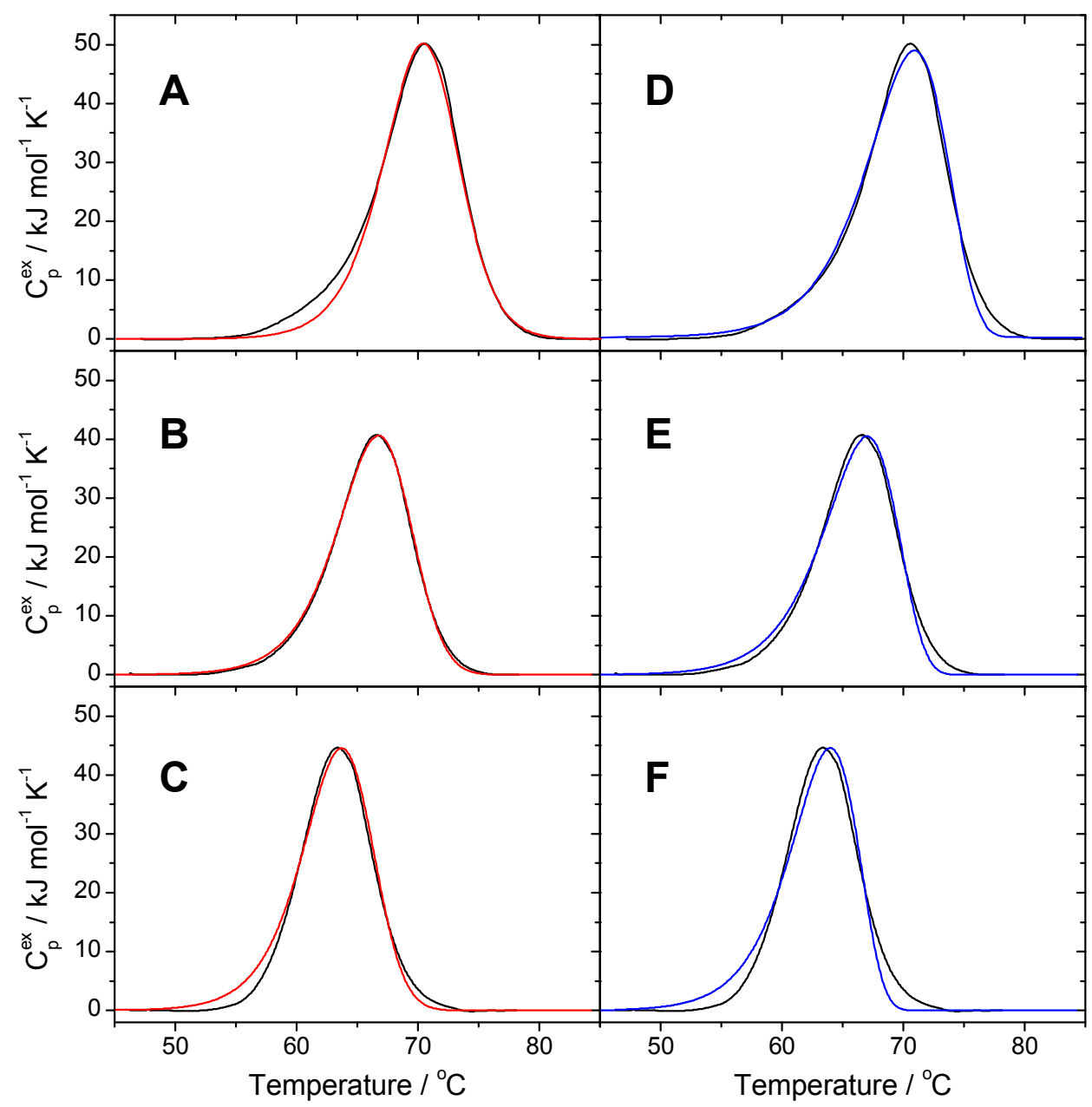

Figure S23. $(\mathbf{A}, \mathbf{B}, \mathbf{C})$ Results of the two-state reversible model fitting (red lines) to the baseline-corrected thermograms (black lines) corresponding to $0.5090,1.0181$ and 1.5271 $\mathrm{mol} \cdot \mathrm{dm}^{-3}$ NEU solutions, respectively. (D) Results of the Lumry-Eyring's irreversible model fitting (blue lines) to the same baseline-corrected thermograms (black lines). 


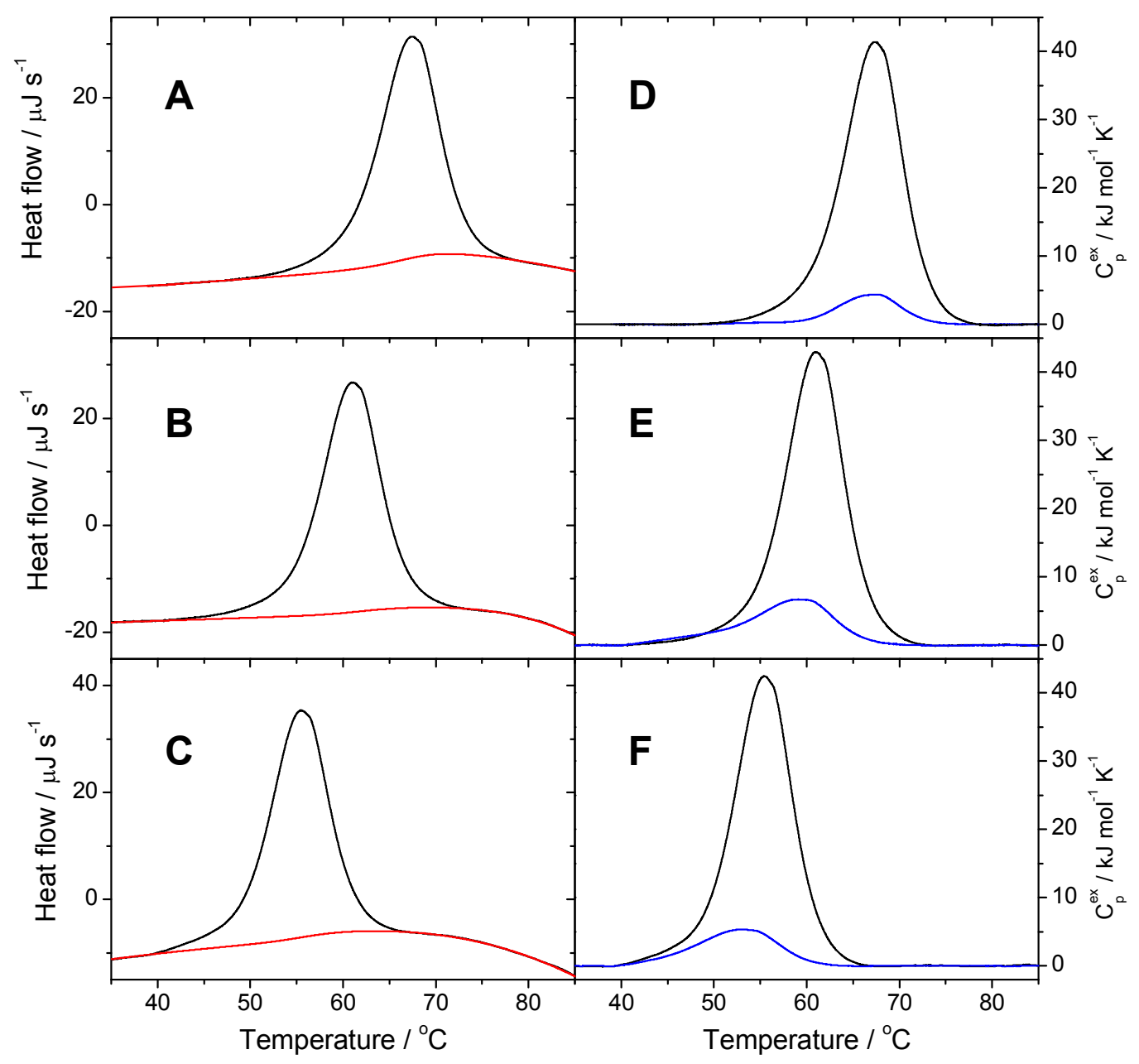

Figure S24. (A,B,C) Thermograms of lysozyme in the presence of DEU at 0.5023, 1.0046 and $1.5069 \mathrm{~mol} \cdot \mathrm{dm}^{-3}$ concentrations, respectively (black lines). Baselines are marked with red lines. (D,E,F) Baseline-corrected thermograms from Figures A,B, and C (black lines) and rescans (blue lines). 


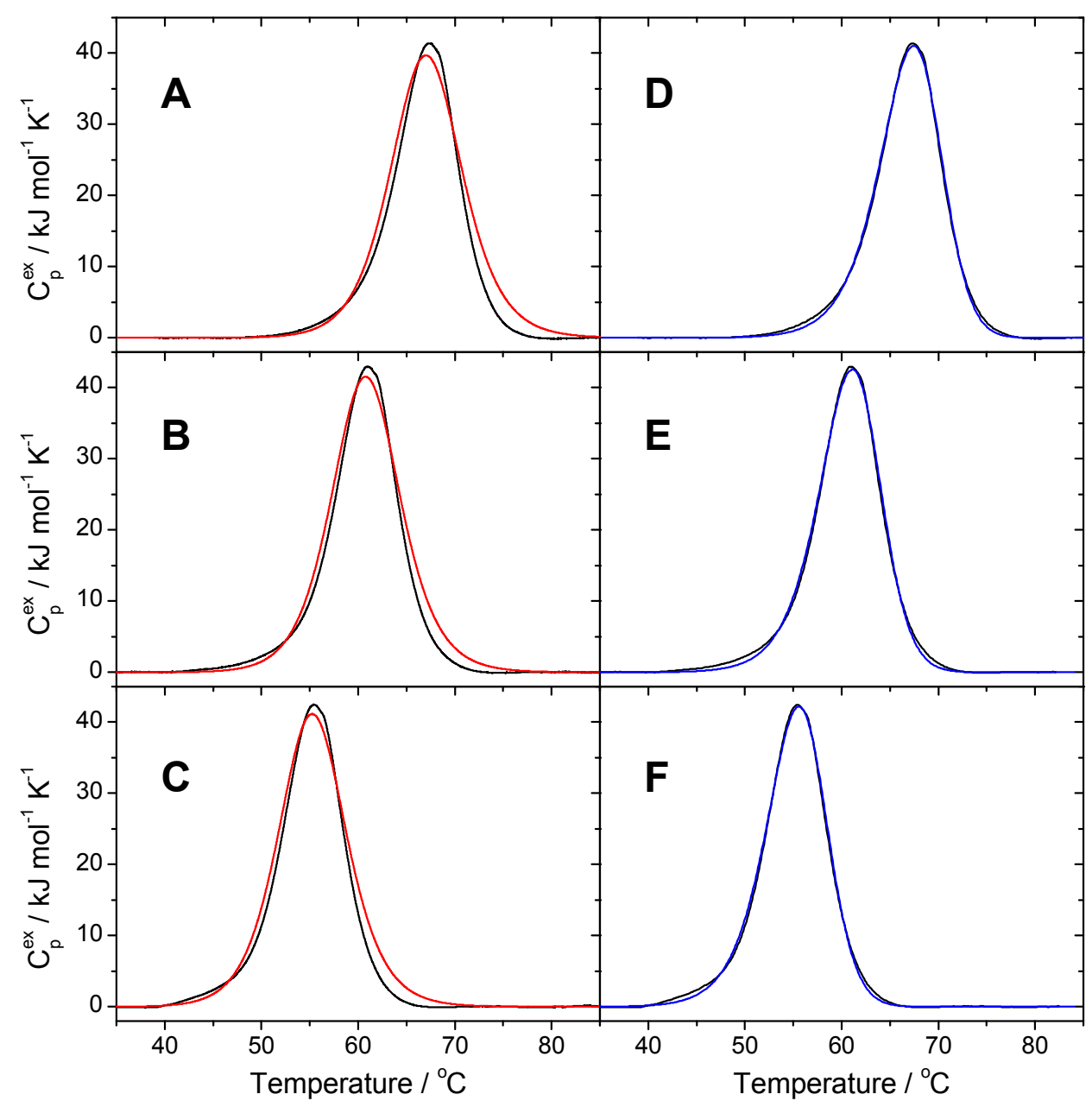

Figure S25. (A,B,C) Results of the two-state reversible model fitting (red lines) to the baseline-corrected thermograms (black lines) corresponding to $0.5023,1.0046$ and 1.5069 $\mathrm{mol} \cdot \mathrm{dm}^{-3}$ DEU solutions, respectively. (D) Results of the Lumry-Eyring's irreversible model fitting (blue lines) to the same baseline-corrected thermograms (black lines). 


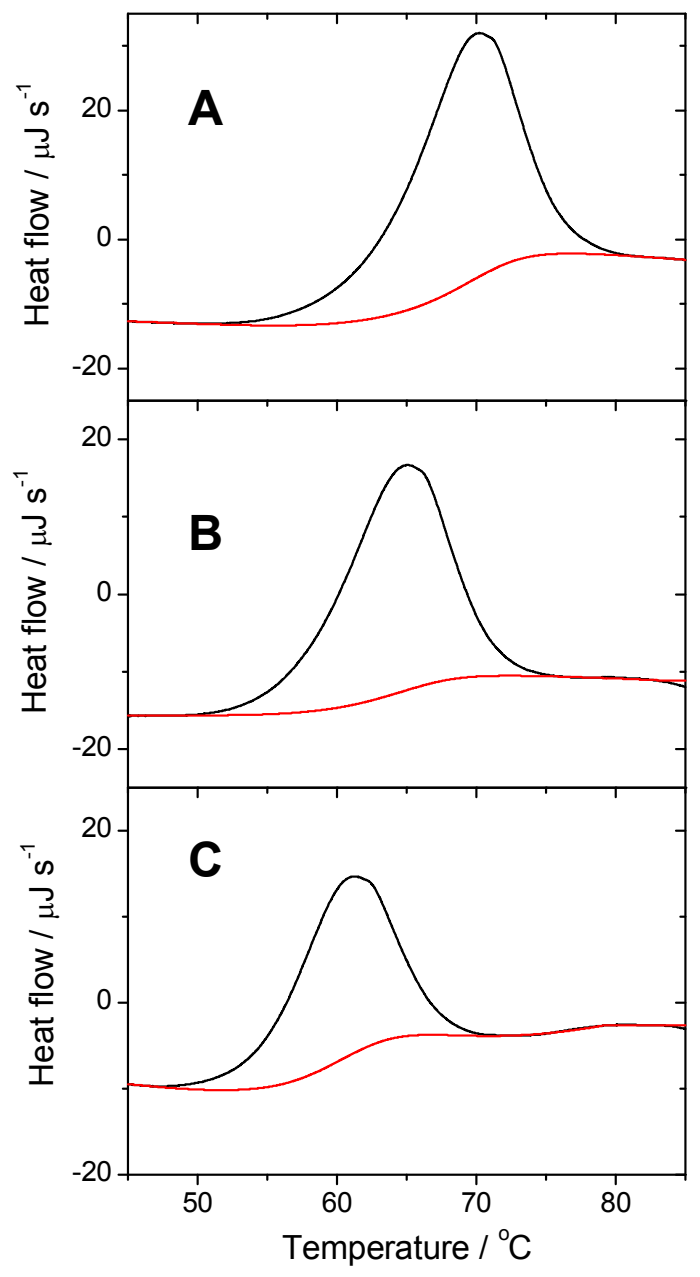

Figure S26. (A,B,C) Thermograms of lysozyme in the presence of NBU at $0.1645,0.3289$ and $0.4934 \mathrm{~mol} \cdot \mathrm{dm}^{-3}$ concentrations, respectively (black lines). Baselines are marked with red lines. 


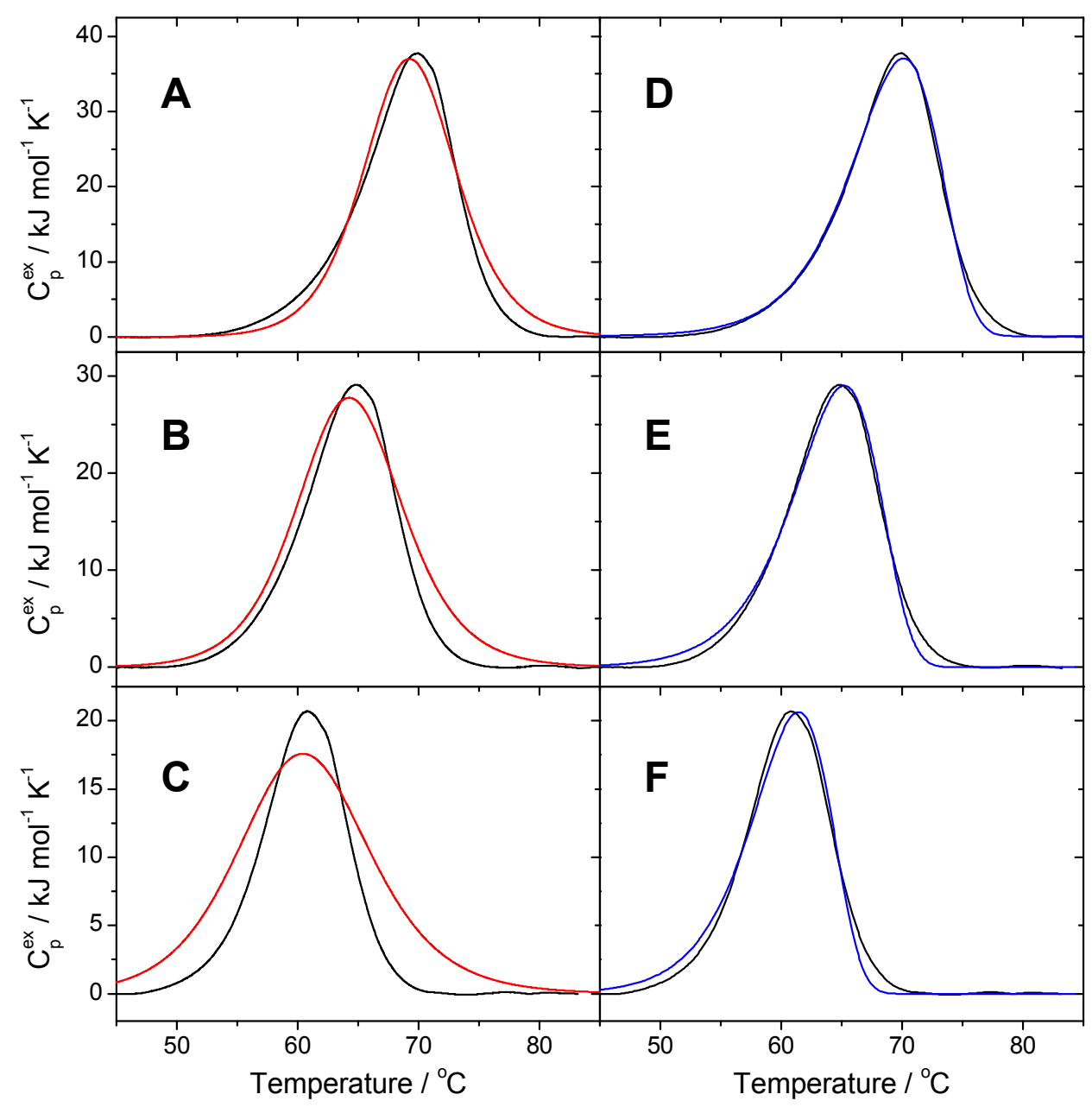

Figure S27. (A,B,C) Results of the two-state reversible model fitting (red lines) to the baseline-corrected thermograms (black lines) corresponding to $0.1645,0.3289$ and 0.4934 $\mathrm{mol} \cdot \mathrm{dm}^{-3}$ NBU solutions, respectively. (D) Results of the "kinetic" irreversible model fitting (blue lines) to the same baseline-corrected thermograms (black lines). 
S3. Difference HDO spectra method: determination and interpretation of the solute affected water spectra.

\section{S3.1. Sample preparation}

Stock solutions were prepared by dissolving weighted amounts of respective solutes in deionized water. The series of solution (less concentrated) were obtained by dilution of weighed amounts of stock solution using deionized water. Next, each of the solutions was divided into two parts. Sample solutions for the HDO spectra were made by adding $\mathrm{D}_{2} \mathrm{O}$ (isotopic purity $99.9 \%$, Aldrich) to one part of the solution in an amount of $4 \%$ (by weight) relative to $\mathrm{H}_{2} \mathrm{O}\left(\mathrm{H}_{2} \mathrm{O}+\mathrm{D}_{2} \mathrm{O}=2 \mathrm{HDO}, K \approx 4\right)$ contained in a solution. The reference solutions (solutes in $\mathrm{H}_{2} \mathrm{O}$ without $\mathrm{D}_{2} \mathrm{O}$ ) were prepared by adding $\mathrm{H}_{2} \mathrm{O}$ to the stock solution in molary amount equivalent to $\mathrm{D}_{2} \mathrm{O}$ addition in solution of the sample.

All solution densities were measured using an Anton Paar DMA 5000 densitometer at $25.000 \pm 0.001{ }^{\circ} \mathrm{C}$.

\section{S3.2. The method}

The method is based on the assumption that it is possible to divide the water population in solution into two additive contributions: the "bulk" water and the solute-affected water. These contributions corresponds to the spectrum of "bulk" water identical to the pure water spectrum, $\varepsilon_{b}$, and the solute-affected water spectrum, $\varepsilon_{a}$, respectively. The solute-affected water spectrum extrapolated to the infinite dilution, $\varepsilon_{a}$, can be determined on the basis of is Equation (1):

$$
\varepsilon_{a}=\frac{1}{N M}\left(\frac{\partial \varepsilon}{\partial m}\right)_{m=0}+\varepsilon_{b}
$$


where $N$ parameter is the "affected number", equal to the number of moles of water affected by one mole of solute, $M$ is the mean $\left(4 \% \mathrm{D}_{2} \mathrm{O}\right.$ in $\mathrm{H}_{2} \mathrm{O}$ in the case of $\mathrm{HDO}$ spectra) molar mass of water $\left(\mathrm{kg} \cdot \mathrm{mol}^{-1}\right)$, and $m$ denotes the molality of the solution $\left(\mathrm{mol} \cdot \mathrm{kg}^{-1}\right)$. The derivative $\partial \varepsilon / \partial m$, in the infinite dilution limit, can be calculated as the derivative at $m=0$ of the linear or quadratic regression curve used to fit the $v(m)$ dependency, for each wavelength separately.

In this method, the "trial" solute-affected water spectrum for a given $N$ value is fitted using the baseline, analytical bands, and the bulk water spectrum. The minimal number of analytical bands, which give an adequate fit, is considered as the right number of component bands. The product of Gaussian and Lorentzian peak functions is used as the starting analytical band shape in the band fitting procedure. All parameters (shape, position, intensity and band half-width) are variable during the fit, with the exception of the bulk solvent spectrum, for which only intensity remains variable. The maximum value of $N$, for which the solute-affected solvent spectrum still contains a negligible amount of the bulk solvent spectrum, is considered as the "true" value of $N$ (usually, the threshold value for bulk water contribution is set at $0.5 \%$ of the total integrated intensity of the $\varepsilon_{a}$ spectrum). The $\varepsilon_{a}$ spectrum corresponding to this $N$ is regarded as the "true" affected water spectrum. Thus, both unknowns are obtained simultaneously.

The detailed procedure of spectral data analysis toward extraction of the solute-affected water spectrum was described previously. ${ }^{1-4}$ This method was used extensively in our laboratory inter alia to study the hydration of aqueous solutions of molecules of biological importance such as osmolytes and proteins. ${ }^{5-7}$ The effectiveness of this method has been confirmed by molecular dynamics simulations ${ }^{5,7,8}$ as well as by chemometric analysis of HDO spectra in aqueous solutions. ${ }^{4}$ 


\section{S3.3. Determination of the osmolyte-affected water spectra.}

We performed detailed analysis of the spectra of the solution series of denaturing osmolytes.

An approximation of the experimental spectra $\varepsilon\left(v_{i}\right)$ vs. molality $m$ (including $m=0$ ) at each wavenumber $v_{\mathrm{i}}$ by the least-squares method allows the calculation of the respective derivative. Figure. S28 presents the molar absorptivity derivatives $(\partial \varepsilon / \partial \mathrm{m})_{\mathrm{m}=0}$ obtained on the basis of the measured spectra for the solution series of ureas. We using quadratic regression of $\varepsilon(v)$ values vs. solution molalities for $\mathrm{U}$ and TMU and linear regression for the other alkyl derivatives of urea $\left(\mathrm{R}^{2}>0.9997\right)$. Together with the bulk HDO spectrum, they were used to determine the solute-affected HDO spectra according to Eq. 1 (Main Part) and the procedure of band shape analysis previously described in detail. ${ }^{2}$ The solute-affected spectra are shown in Figure S29. They have been obtained for the following affected numbers $(N)$ : $5.0(\mathrm{U}), 1.8$ (NMU), 2.4 (NEU), 3.1 (NBU), 2.7 (DMU), 3.2 (DEU), 3.8 (TMU). These results suggest that the substitution of one or more hydrogen atoms in urea molecule with an alkyl group reduces the $N$ parameter. Moreover the $N$ gets higher when the number or length of the alkyl substituent increase.

\section{S3.4. Isolation and subtraction of ND bands.}

The abovementioned osmolyte-affected spectra (Figure S29) possess a small contribution of ND vibration band $v(N D)$ if the osmolyte has a possibility to exchange a proton from the $\mathrm{NH}$ group (present in almost every urea derivative molecule). To subtract the $v(N D)$ contribution, necessary to obtain a reliable $P\left(R_{o o}\right)$ distribution function, the spectrum of TMUaffected HDO water is exploited. An osmolyte-affected spectrum, obtained by the method described in the paper, is again fitted with the TMU-affected HDO band. All parameters of this band were allowed to change during fitting procedure (position, height, half-height 
width), except the shape. The residual was fitted with analytical bands, which then received the physical meaning (see Figure S30).

To identify which of these bands can be ascribed to the ND stretching vibrations, $v(N D)$, theoretical calculations in the DFT framework were performed with B3LYP hybrid functional and the $6-311++\mathrm{G}(\mathrm{d}, \mathrm{p})$ basis set. All calculations were carried out within the GAUSIAN 03 software package. A proper conformer (cis or trans) was selected for calculations on the basis of the cis $<->$ trans equilibrium constant: cis for NMU and NEU, trans for NBU, and cis-cis for DMU and DEU. Structures of the compounds are presented in Figure S31. The comparison between DFT-calculated and experimentally isolated $v(N D)$ band parameters are presented in Table S5. The experimental values generally agree with the calculated ones. In the case of the latter ones the positions of ND bands are only slightly shifted to higher values.

In the case of HDO spectra in the presence of monosubstituted urea derivatives (NMU, NEU, NBU) two sub-bands can be attributed to the ND vibrations: ca. $2440 \mathrm{~cm}^{-1}$ and ca. 2535 $\mathrm{cm}^{-1}$. The first one corresponds to the vibration of ND bond in the direct neighborhood of alkyl substituent (position 1 in Figure S31). The seconds one, corresponding to the amino protons of the unsubstituted amino group (positions 2 and 3 in Figure S31), can be interpreted similarly to the case of urea ND bands (i.e. two inseparable sub-bands). Double substitution of urea molecule (in 1,3 positions) results in one ND band at $2470 \mathrm{~cm}^{-1}$ or $2493 \mathrm{~cm}^{-1}$ for DMU or DEU, respectively.

It should be noted that HDO spectrum in the presence of urea includes a weak component band at $2631 \mathrm{~cm}^{-1}$, which is due to a water molecule weakly interacting with the hydrogen atom of the amino group. ${ }^{5,6,9}$ The weak OD band is not visible in HDO spectra corresponding to urea derivatives. We believe that this results from the presence of alkyl 
substituent, which has the ability to organize water molecules into clathrate-like cage around amino protons of urea derivatives. Protons of disubstituted alkyl derivatives are undoubtedly closed in such a cage and avoid direct interactions with water. In the case of a monosubstituted urea derivative the amino proton closer to the alkyl substituent is closed in the cage, and cannot interact with surrounding water molecules, while protons of the second amino group are either closed in a large clathrate cage, or engaged in even weaker hydrogen bonds with water molecules of the cage. Our results, concerning urea ${ }^{5}$ and amides, ${ }^{8}$ allow us to speculate that the clathrate-like net of hydrogen bonds around non-polar groups and a proton can be energetically even more favorable than a net surrounding only the non-polar group.

Precise analysis of the affected HDO spectra corresponding to urea derivatives solutions reveals that an additional very weak sub-band appears between 2394 and $2425 \mathrm{~cm}^{-1}$. The band does not have any physical meaning and can be treated as a correction of the band shape of the main HDO band, necessary at the fitting step of the band-shape analysis.

After the determination of the ND bands positions, the identified bands from the osmolyte-affected HDO spectra can be subtracted. The pure osmolyte-affected HDO spectra are presented in Figure 3a (Main Part).

\section{S3.5. Structural and energetic characterization of water affected by osmolytes.}

The position of the OD water band has a direct relationship with the energy of the hydrogen bonds. On the basis of the Badger-Bauer's rule, ${ }^{10}$ which states that water hydrogenbond energy changes linearly with the shift in $\mathrm{OH}(\mathrm{OD})$ band position, it is possible to define the energetic state of water molecules in hydration spheres of osmolytes. The position of the HDO absorption band maximum $\left(v^{\circ}\right.$ OD $)$ refers to the most probable hydrogen bond energy, and the position of the band gravity center $\left(v^{\mathrm{g}} \mathrm{OD}\right)$ relates to the mean energy of water 
hydrogen bonds. Values of $v^{\mathrm{g}}$ OD can be later on used to determine the average change of the hydrogen bond energy per one mole of affected water $-\Delta \mathrm{E}_{\mathrm{H}-\mathrm{B}}^{\mathrm{g}}-$ according to the procedure described previously. ${ }^{11}$

Band shapes of OD water spectra, can be transformed into the oxygen-oxygen distance distribution function $\mathrm{P}\left(\mathrm{R}_{\mathrm{OO}}\right)$ of the water molecules according to Equation (2):

$$
\mathrm{R}_{\mathrm{OO}}=\left[16.01-\ln \left(2727-v_{\mathrm{OD}}\right)\right] / 3.73
$$

with $\mathrm{R}_{\mathrm{OO}}$ expressed in $\AA$ and $v_{\mathrm{OD}}$ in $\mathrm{cm}^{-1} \cdot 12,13$

The oxygen-oxygen distance distribution function $P\left(R_{O O}\right)$ include also the contribution of $\mathrm{N}^{\cdots} \mathrm{O}$ distances to the interaction of water molecule with amino group, and $\mathrm{O} \cdots \mathrm{O}$ distances in the case of interaction with the carbonyl oxygen of urea and its alkyl derivatives. 
Table S5. Experimental and theoretical values of $v(N D)$ band positions.

\begin{tabular}{cll}
\hline & ${v_{N D}}^{a}$ & $\nu_{N D}{ }^{(c)}$ \\
\hline \multirow{2}{*}{ NMU } & 2451 & $2480^{(1)}$ \\
& 2541 & $2558^{(2)}$ \\
& & $2578^{(3)}$ \\
\hline \multirow{2}{*}{ NEU } & 2432 & $2484^{(1)}$ \\
& 2534 & $2559^{(2)}$ \\
& & $2576^{(3)}$ \\
\hline \multirow{2}{*}{ NBU } & 2438 & $2472^{(1)}$ \\
& 2530 & $2589^{(2)}$ \\
& & $2558^{(3)}$ \\
\hline DMU & 2470 & $2483^{(1)}$ \\
& & $2481^{(2)}$ \\
\hline DEU & 2493 & $2465^{(1)}$ \\
& & $2458^{(2)}$ \\
\hline
\end{tabular}

${ }^{a}$ Experimental values of $v(N D)$ band position $\left(\mathrm{cm}^{-1}\right)$ (see Figure S30). ${ }^{b}$ Calculated (DFT method, PCM model) values of $v(N D)$ band position $\left(\mathrm{cm}^{-1}\right)$ for the structures of alkylsubstituted ureas shown in Figure S31; ${ }^{c}$ Numbers in the brackets are shown in Figure S31. 
Table S6. The parameters of HDO bands of water affected by stabilizing osmolytes, and the respective intermolecular oxygen - oxygen distances. $R_{O O}$ errors have been estimated on the basis of the HDO bands position errors.

\begin{tabular}{ccccccccc}
\hline solute & $N^{a}$ & $v_{O D}{ }^{b}$ & $v_{O D}^{g}{ }^{c}$ & $f w h h^{d}$ & $I^{e}$ & $R_{O O}^{o}$ & $R_{O O^{g}}^{g}$ & $\Delta^{g}{ }^{g}{ }_{H-B}^{h}$ \\
\hline $\mathrm{GLY}^{i}$ & $11.0 \pm 0.5$ & $2505 \pm 2$ & $2495 \pm 2$ & $185 \pm 4$ & 9986 & $2.821 \pm 0.003$ & $2.831 \pm 0.003$ & -2.0 \\
$\mathrm{ALA}^{i}$ & $11.9 \pm 0.5$ & $2500 \pm 2$ & $2490 \pm 2$ & $183 \pm 4$ & 8530 & $2.819 \pm 0.003$ & $2.827 \pm 0.003$ & -3.0 \\
$\mathrm{PRO}^{i}$ & $11.2 \pm 0.5$ & $2505 \pm 2$ & $2494 \pm 2$ & $185 \pm 4$ & 9114 & $2.819 \pm 0.003$ & $2.829 \pm 0.003$ & -2.2 \\
$\mathrm{VAL}^{i}$ & $8.9 \pm 0.5$ & $2496 \pm 2$ & $2484 \pm 2$ & $184 \pm 4$ & 9077 & $2.809 \pm 0.003$ & $2.819 \pm 0.003$ & -4.2 \\
$\mathrm{ILE}^{i}$ & $10.2 \pm 0.5$ & $2496 \pm 2$ & $2482 \pm 2$ & $178 \pm 4$ & 7929 & $2.809 \pm 0.003$ & $2.819 \pm 0.003$ & -4.6 \\
$\mathrm{PHE}^{i}$ & $16.0 \pm 0.5$ & $2502 \pm 2$ & $2488 \pm 2$ & $184 \pm 4$ & 6738 & $2.809 \pm 0.003$ & $2.824 \pm 0.003$ & -3.4 \\
$\mathrm{NMG}^{j}$ & $5.0 \pm 0.5$ & $2457 \pm 2$ & $2476 \pm 2$ & $224 \pm 4$ & 7032 & $2.767 \pm 0.003$ & $2.813 \pm 0.003$ & -5.7 \\
$\mathrm{DMG}^{j}$ & $4.8 \pm 0.5$ & $2515 \pm 2$ & $2488 \pm 2$ & $207 \pm 4$ & 7846 & $2.785 \pm 0.003$ & $2.826 \pm 0.003$ & -3.4 \\
$\mathrm{TMG}^{j}$ & $4.0 \pm 0.5$ & $2522 \pm 2$ & $2492 \pm 2$ & $202 \pm 4$ & 11994 & $2.800 \pm 0.003$ & $2.828 \pm 0.003$ & -2.6 \\
$\mathrm{TMAO}^{i}$ & $8.5 \pm 0.5$ & $2495 \pm 2$ & $2470 \pm 2$ & $196 \pm 4$ & 11339 & $2.810 \pm 0.003$ & $2.805 \pm 0.003$ & -6.9 \\
\hline
\end{tabular}

${ }^{a}$ Affected number. ${ }^{b}$ Band position at maximum $\left(\mathrm{cm}^{-1}\right) \cdot{ }^{c}$ Band position at gravity center $\left(\mathrm{cm}^{-}\right.$ $\left.{ }^{1}\right){ }^{d}$ Full width at half-height $\left(\mathrm{cm}^{-1}\right) .{ }^{e}$ Integrated intensity $\left(\mathrm{dm}^{3} \cdot \mathrm{mol}^{-1} \cdot \mathrm{cm}^{-1}\right) .{ }^{f}$ The most probable $\mathrm{O} \cdots \mathrm{O}$ distance $(\AA) .{ }^{g}$ Mean $\mathrm{O} \cdots \mathrm{O}$ distance $(\AA) .{ }^{h}$ The average change of the energy of hydrogen bonds per one mol of the affected water (kJ). ${ }^{i}$ Ref. $9 .{ }^{j}$ Ref. $14 .{ }^{k}$ Ref. 5. 
Table S7. The contributions of vanishing weak hydrogen bonds population in the hydration shell of solutes and solvent-accessible surface of stabilizing osmolytes.

\begin{tabular}{ccccc}
\hline Solute & $\alpha^{a}$ & $\Delta \alpha^{b}$ & $\beta^{c}$ & $\Delta \alpha / \beta \cdot 10^{-3 d}$ \\
\hline $\mathrm{GLY}^{e}$ & 0.055 & - & - & - \\
$\mathrm{ALA}^{e}$ & 0.077 & 0.023 & 24.14 & 0.933 \\
$\mathrm{PRO}^{e}$ & 0.065 & 0.010 & 70.48 & 0.143 \\
$\mathrm{VAL}^{e}$ & 0.106 & 0.051 & 75.91 & 0.678 \\
$\mathrm{ILE}^{e}$ & 0.112 & 0.057 & 101.64 & 0.561 \\
$\mathrm{PHE}^{e}$ & 0.090 & 0.036 & 142.29 & 0.250 \\
$\mathrm{NMG}^{f}$ & 0.139 & 0.084 & 37.39 & 2.258 \\
$\mathrm{DMG}^{f}$ & 0.087 & 0.032 & 63.11 & 0.514 \\
$\mathrm{TMG}^{f}$ & 0.087 & 0.032 & 79.3 & 0.402 \\
$\mathrm{TMAO}^{g}$ & 0.173 & - & - & - \\
\hline
\end{tabular}

${ }^{a}$ The parameter which reflects the contribution of vanishing weak hydrogen bonds of water molecules. The $\alpha$ parameter was calculated as an area (see Figure. $4 \mathrm{~b}$ in Main Part and text for details). ${ }^{b}$ The $\alpha$ parameter for osmolytes relative to glycine $\left(\alpha-\alpha_{G L Y}\right)$, determining the contribution of vanishing weak hydrogen bonds of water molecules around the amino acid side chain and methyl groups of glycine derivatives. ${ }^{c}$ The solvent-accessible surface for the amino acid side chain and methyl groups of glycine derivatives $\left(\AA^{2}\right) .{ }^{d}$ The contribution of vanishing population of weak hydrogen bonds of water molecules per unit surface of the a amino acid side chain and methyl groups of glycine derivatives $\left(\AA^{-2}\right)$. Values of the $\alpha$ parameter are calculated on the basis of the differences in the intermolecular distance distribution function $\Delta P\left(R_{\mathrm{OO}}\right)$ in the range $3.4-2.6 \AA$ as shown in: ${ }^{e}$ Ref. $9,{ }^{f}$ Ref. 14 and ${ }^{g}$ Ref. 5. 


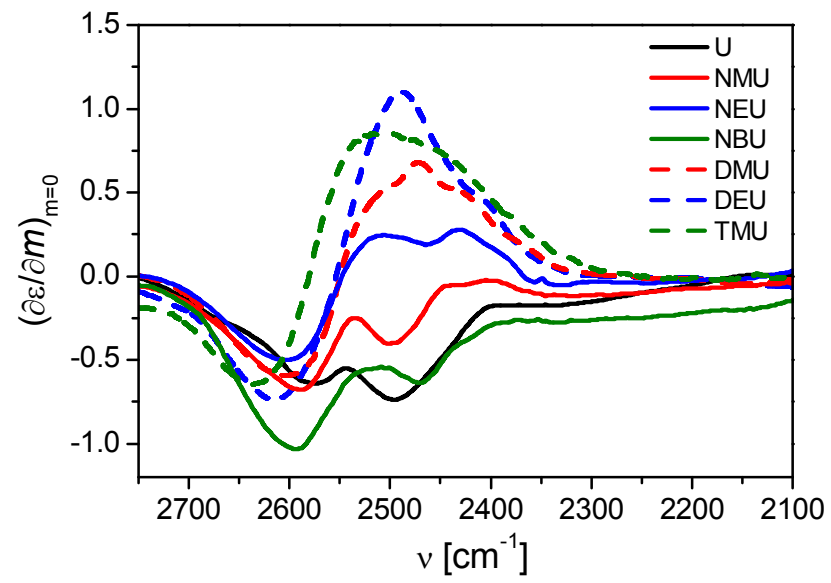

Figure S28. Derivatives at infinite dilution limit $(d \varepsilon / d m)_{m=0}$ for solute-affected HDO spectra in the OD stretching region for solutions of urea and alkyl derivatives of urea. 


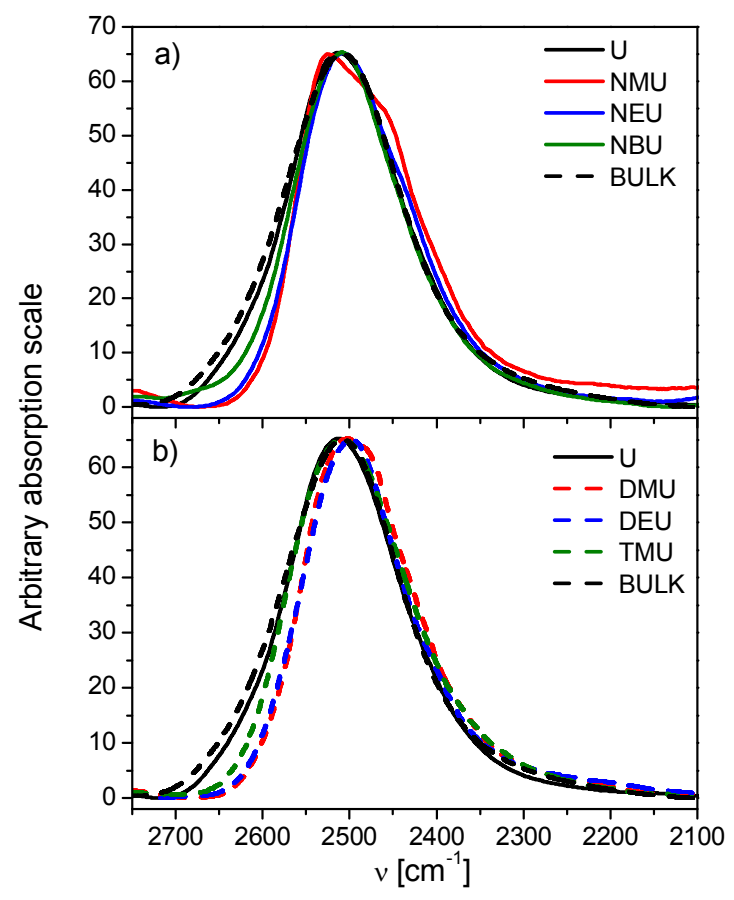

Figure S29. Solute-affected spectra in the OD stretching region for (a) urea and its monosubstituded alkylderivatives and (b) di- and tetra-substituded alkylderivatives of urea together with the bulk HDO spectrum. The spectra have been scaled to the same maximum absorption value for better comparison. 

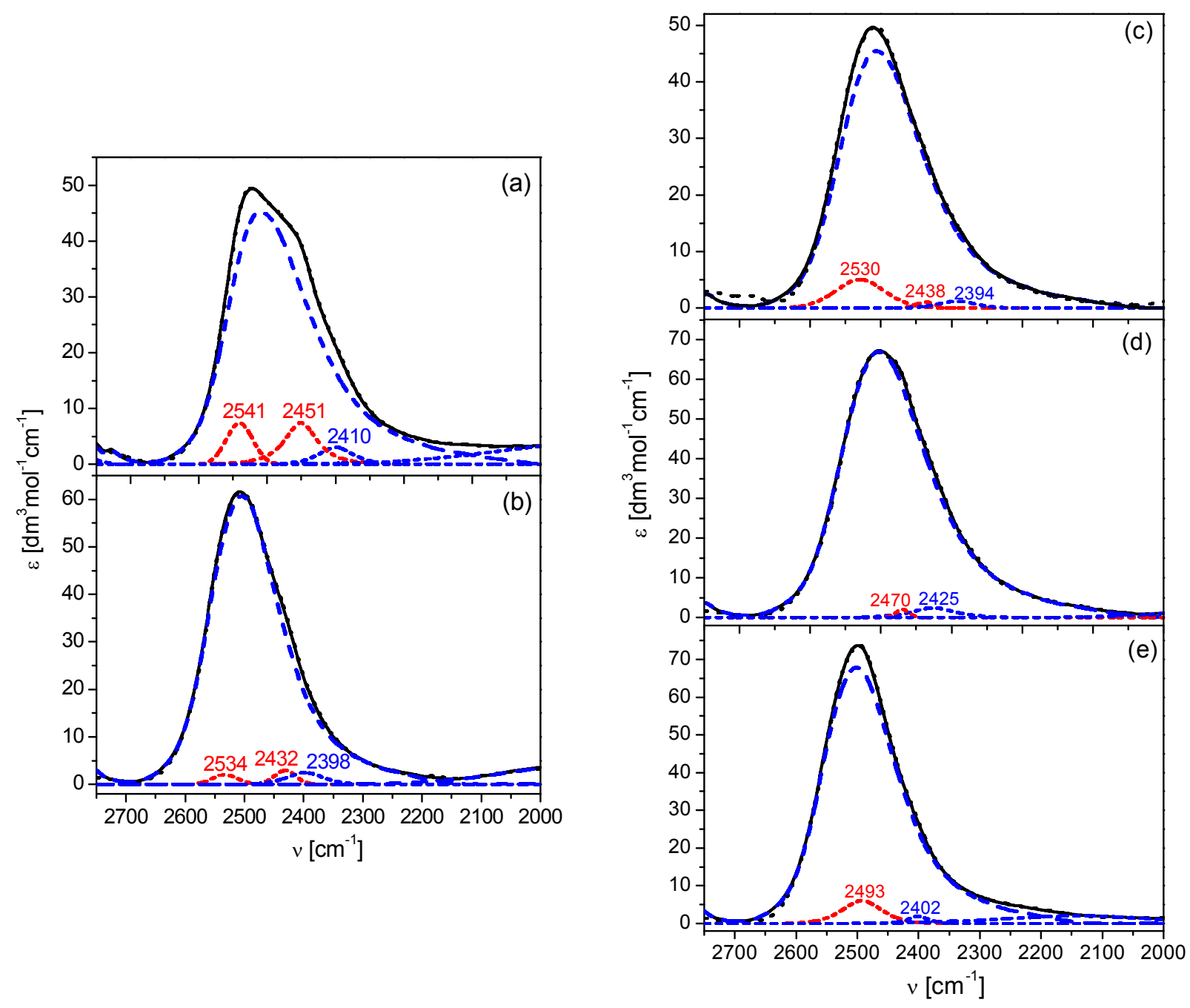

Figure S30. Decomposition of affected spectra in the OD stretching region of HDO for (a) NMU, (b) NEU, (c) NBU, (d) DMU, and (e) DEU into component bands. Solid black line: original affected spectrum; dotted black line: sum of the component bands (covered by the solid line of the original spectrum); dashed blue line: OD component bands; dashed red line: ND component bands. 
<smiles>C=C(C)C(I)C(C)C(C)C</smiles>

NMU<smiles>[Z]C(C)C(C)C(I)C(C)C(=O)C(=O)O</smiles>

NEU

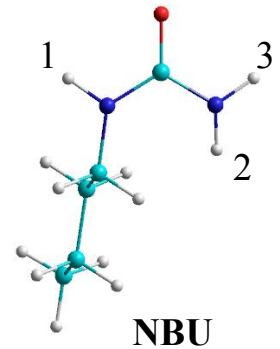

NBU<smiles>CC(C)C(C)C(C)C(C)C(C)C(C)C</smiles>

DMU

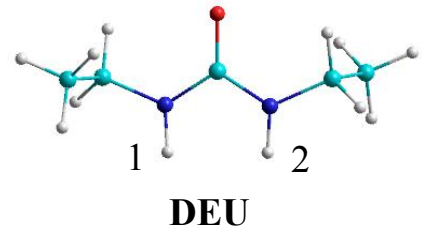

DEU

Figure S31. Structure of alkyl derivatives of urea used for the calculation of ND vibrations, calculated at the B3LYP/6-311++G(d,p) level with the continuum model (PCM). The calculated results are presented in Table S5. 


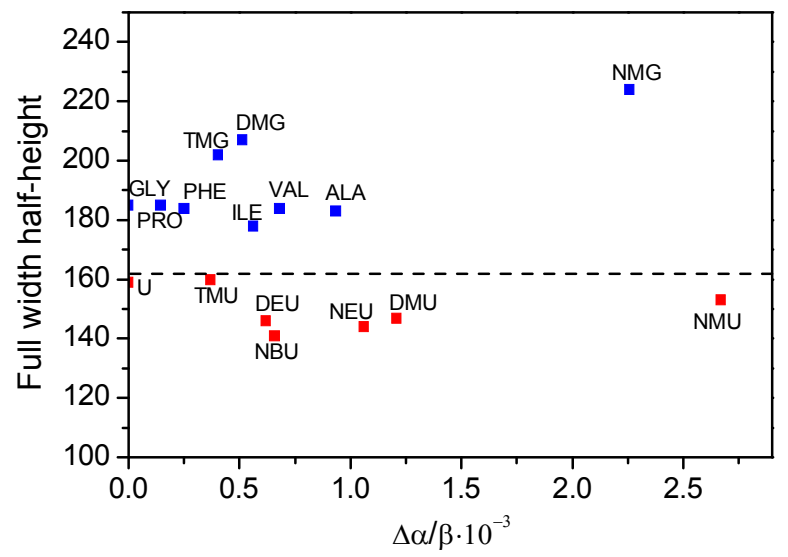

Figure S32. Correlation of full-width half-height ( $f w h h)$ of the OD band (values are taken from Table S6 for stabilizing osmolytes and Table 2 in Main Part for denaturing osmolytes) and the vanishing population of weak hydrogen bonds of water molecules per unit surface of the alkyl chain of urea and its derivatives, amino acid side chain and methyl groups of glycine derivatives (values are taken from Table S7 for stabilizing osmolytes and Table 3 in Main Part for denaturing osmolytes), $\Delta \alpha / \beta$. The dashed horizontal line denotes the full width halfheight $(f w h h)$ of the OD band for "bulk" water $\left(162 \mathrm{~cm}^{-1}\right)$. 


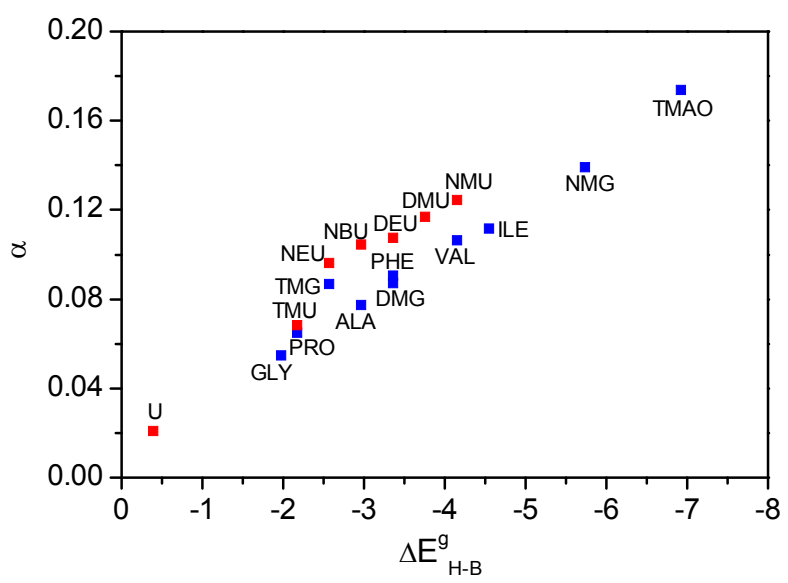

Figure S33. Correlation of the contributions of vanishing population of weak hydrogen bonds of water molecules $(\alpha)$ and the average change of the energy of hydrogen bonds for one mol of the affected water $\left(\Delta \mathrm{E}_{\mathrm{H}-\mathrm{B}}^{\mathrm{g}}\right)$ by denaturing (values are taken from Table 2 and Table 3 in Main Part) and stabilizing osmolytes (values are taken from Table S6 and Table S7). 

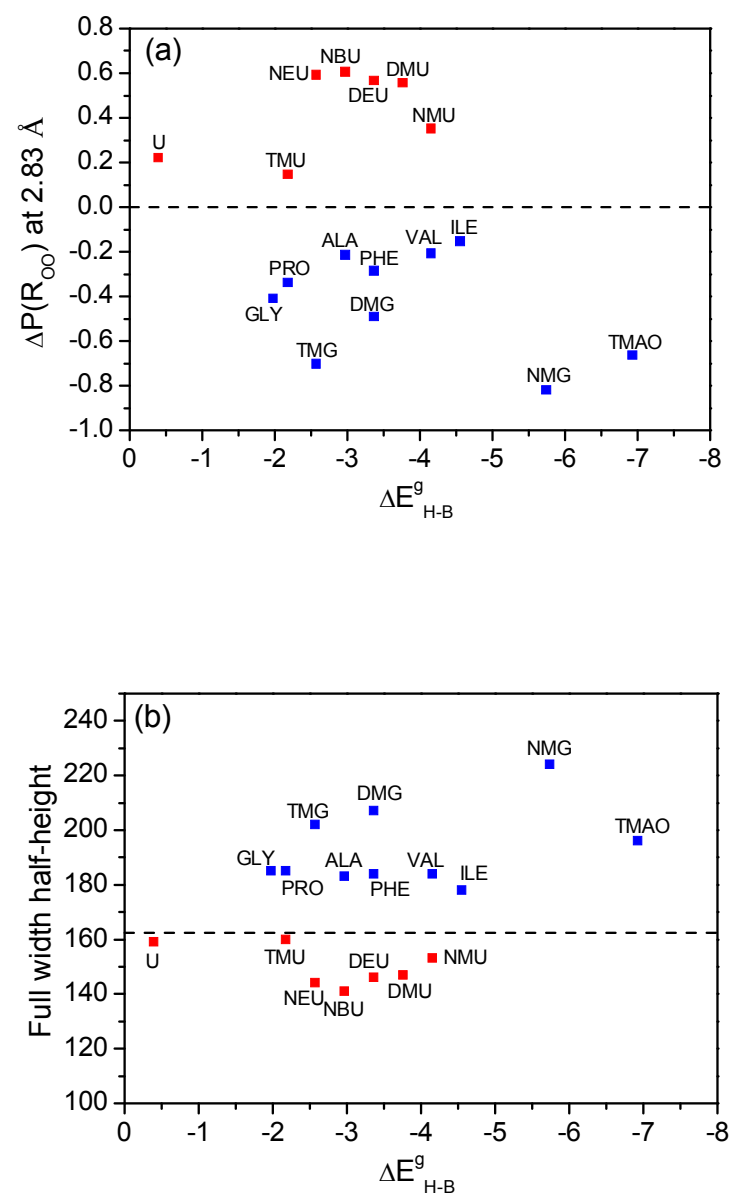

Figure S34. (a) Correlation of values of $\Delta P\left(R_{O O}\right)$ at the most probable water-water distance $(2.83 \AA)$ and the average change of the energy of hydrogen bonds for one mol of the affected water $\left(\Delta \mathrm{E}_{\mathrm{H}-\mathrm{B}}^{\mathrm{g}}\right)$ by denaturing (values are taken from Table 2 in Main Part) and stabilizing osmolytes (values are taken from Table S6). (b) Correlation of full-width half-height ( $f w h h$ ) of the OD band (values are taken from Table S6 for stabilizing osmolytes and Table 2 in Main Part for denaturing osmolytes) and the average change of the energy of hydrogen bonds for one mol of the affected water $\left(\Delta \mathrm{E}_{\mathrm{H}-\mathrm{B}}^{\mathrm{g}}\right)$ by denaturing and stabilizing osmolytes. The dashed horizontal line denotes the full width half-height $(f w h h)$ of the OD band for "bulk" water (162 $\left.\mathrm{cm}^{-1}\right)$. 


\section{REFERENCES}

(1) Stangret, J. Solute-Affected Vibrational-Spectra of Water in $\mathrm{Ca}\left(\mathrm{ClO}_{4}\right)_{2}$ Aqueous Solutions. Spectrosc. Lett. 1988, 21, 369-381.

(2) Śmiechowski, M.; Stangret, J. Vibrational Spectroscopy of Semiheavy Water (HDO) as a Probe of Solute Hydration. Pure Appl. Chem. 2010, 82, 1869-1887.

(3) Bruździak, P.; Rakowska, P. W.; Stangret, J. Chemometric Method of Spectra Analysis Leading to Isolation of Lysozyme and CtDNA Spectra Affected by Osmolytes. Appl. Spectrosc. 2012, 66, 1302-1310.

(4) Bruździak, P.; Panuszko, A.; Stangret, J. Chemometric Determination of SoluteAffected Solvent Vibrational Spectra as a Superior Way of Information Extraction on Solute Solvation Phenomena. Vib. Spectrosc. 2010, 54, 65-71.

(5) Panuszko, A.; Bruździak, P.; Zielkiewicz, J.; Wyrzykowski, D.; Stangret, J. Effects of Urea and Trimethylamine-N-oxide on the Properties of Water and the Secondary Structure of Hen Egg White Lysozyme. J. Phys. Chem. B 2009, 113, 14797-14809.

(6) Panuszko, A.; Śmiechowski, M.; Stangret, J. Fourier Transform Infrared Spectroscopic and Theoretical Study of Water Interactions with Glycine and its NMethylated Derivatives. J. Chem. Phys. 2011, 134, 115104(1)-115104(9).

(7) Panuszko, A.; Wojciechowski, M.; Bruździak, P.; Rakowska, P. W.; Stangret, J. Characteristics of Hydration Water around Hen Egg Lysozyme as the Protein Model in Aqueous Solution. FTIR Spectroscopy and Molecular Dynamics Simulation. Phys. Chem. Chem. Phys. 2012, 14, 15765-15773.

(8) Panuszko, A.; Gojlo, E.; Zielkiewicz, J.; Smiechowski, M.; Krakowiak, J.; Stangret, J. Hydration of Simple Amides. FTIR Spectra of HDO and Theoretical Studies. J. Phys. Chem. B 2008, 112, 2483-2493.

(9) Panuszko, A.; Adamczak, B.; Czub, J.; Gojło, E.; Stangret, J. Hydration of Amino Acids: FTIR Spectra and Molecular Dynamics Studies. Amino Acids 2015, 47, 2265 2278.

(10) Badger, R. M.; Bauer, S. H. Spectroscopic Studies of the Hydrogen Bond. II. The Shift of the O-H Vibrational Frequency in the Formation of the Hydrogen Bond. J. Chem. Phys. 1937, 5, 839-851. 
(11) Stangret, J.; Kamieńska-Piotrowicz, E. Effect of Tetraphenylphosphonium and Tetraphenylborate Ions on the Water Structure in Aqueous Solutions; FTIR Studies of HDO Spectra. J. Chem. Soc. Faraday Trans. 1997, 93, 3463-3466.

(12) Berglund, B.; Lindgren, J.; Tegenfeldt, J. O-H and O-D Stretching Vibrations in Isotopically Dilute HDO Molecules in Some Solid Hydrates. J. Mol. Struct. 1978, 43, 169-177.

(13) Bratos, S.; Leicknam, J. C.; Pommeret, S. Relation Between the OH Stretching Frequency and the OO Distance in Time-Resolved Infrared Spectroscopy of Hydrogen Bonding. Chem. Phys. 2009, 359, 53-57.

(14) Bruździak, P.; Panuszko, A.; Stangret, J. Influence of Osmolytes on Protein and Water Structure: A Step To Understanding the Mechanism of Protein Stabilization. J. Phys. Chem. B 2013, 117, 11502-11508. 